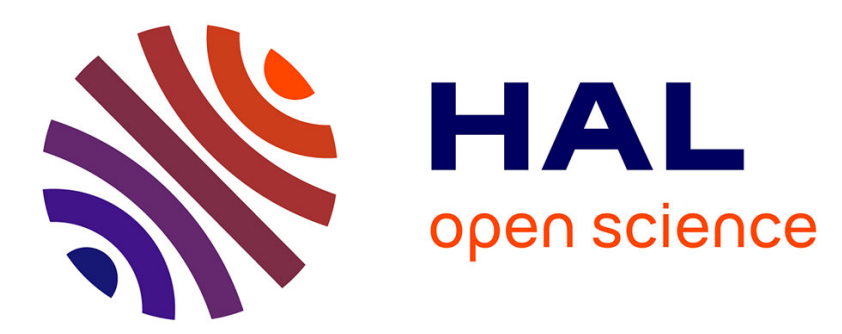

\title{
Adaptive Estimation for Uncertain Nonlinear Systems with Measurement Noise: A Sliding-Mode Observer Approach
}

Roberto Franco, Héctor Ríos, Denis Efimov, Wilfrid Perruquetti

\section{- To cite this version:}

Roberto Franco, Héctor Ríos, Denis Efimov, Wilfrid Perruquetti. Adaptive Estimation for Uncertain Nonlinear Systems with Measurement Noise: A Sliding-Mode Observer Approach. International Journal of Robust and Nonlinear Control, 2020, 10.1002/rnc.5220 . hal-02912951

\section{HAL Id: hal-02912951 \\ https://hal.inria.fr/hal-02912951}

Submitted on 7 Aug 2020

HAL is a multi-disciplinary open access archive for the deposit and dissemination of scientific research documents, whether they are published or not. The documents may come from teaching and research institutions in France or abroad, or from public or private research centers.
L'archive ouverte pluridisciplinaire HAL, est destinée au dépôt et à la diffusion de documents scientifiques de niveau recherche, publiés ou non, émanant des établissements d'enseignement et de recherche français ou étrangers, des laboratoires publics ou privés. 
DOI: $\mathrm{xxx} / \mathrm{xxxx}$

\title{
ARTICLE TYPE
}

\section{Adaptive Estimation for Uncertain Nonlinear Systems with Measurement Noise: A Sliding-Mode Observer Approach $\|^{\dagger}$}

\author{
Roberto Franco ${ }^{1}$ | Héctor Ríos*,1,2 | Denis Efimov ${ }^{3,4}$ | Wilfrid Perruquetti ${ }^{5}$
}

\author{
${ }^{1}$ Tecnológico Nacional de México/I.T. La \\ Laguna, División de Estudios de Posgrado \\ e Investigación, Torreón, Coahuila, México \\ ${ }^{2}$ Cátedras CONACYT, Ciudad de México, \\ México \\ ${ }^{3}$ Inria, Univ. Lille, CNRS, UMR 9189 - \\ CRIStAL, F-59000, Lille, France \\ ${ }^{4}$ Department of Control Systems and \\ Informatics, Information Technologies \\ Mechanics and Optics University, Saint \\ Petersburg 197101, Russia \\ ${ }^{5}$ Centrale Lille, CNRS, UMR \\ 9189-CRIStAL, 59655 Villeneuve d'Ascq, \\ France
}

\section{Correspondence}

*Héctor Ríos Email:

hriosb@correo.itlalaguna.edu.mx

Present Address

hriosb@correo.itlalaguna.edu.mx

\begin{abstract}
Summary
This paper deals with the problem of adaptive estimation, i.e. the simultaneous estimation of the state and time-varying parameters, in the presence of measurement noise and state disturbances, for a class of uncertain nonlinear systems. An adaptive observer is proposed based on a nonlinear time-varying parameter identification algorithm and a sliding-mode observer. The nonlinear time-varying parameter identification algorithm provides a fixed-time rate of convergence, to a neighborhood of the origin, while the sliding-mode observer ensures ultimate boundedness for the state estimation error attenuating the effects of the external disturbances. Linear matrix inequalities are provided for the synthesis of the adaptive observer while the convergence proofs are given based on the Lyapunov and Input-to-State Stability theory. Finally, some simulation results show the feasibility of the proposed approach.
\end{abstract}

\section{KEYWORDS:}

Adaptive Observer; Nonlinear Systems; Sliding-Modes

\section{1 | INTRODUCTION}

Disturbances and uncertainties widely exist in almost all real world scenarios, in the form of external perturbations, unknown system dynamics and/or unknown parameters. There are two main approaches to counteract the perturbations in control system applications: robust and adaptive control. The former one aims at a fixed design that is able to tolerate all perturbations in a given class, while the latter method introduces additional loops to compensate the uncertainty of a chosen class. Last years, the theory of adaptive control has grown to turn into one of the widest domains in terms of algorithms, analytical tools and techniques for design (see, for instance ${ }^{2}$ and ${ }^{3}$ ).

Particularly, the design of observers estimating simultaneously the whole state and the parameters of the system by some on-line adaptation law is an important problem in the adaptive control area (see, e.g. ${ }^{4}$ ). In this framework, a class of adaptive extended state observers is proposed in ${ }^{5}$ that significantly increases the applicability of state observers to nonlinear disturbed systems. $\mathrm{In}[\mathrm{6}$ the problem of adaptive observer synthesis for Lipschitz nonlinear systems is addressed. The sufficient conditions are given in terms of Linear Matrix Inequalities (LMIs) that ensure the state estimation error convergence to zero with known parameters. Also, an adaptive observer form is presented which is used to achieve the adaptive estimation under additional constraints. In this vein, in ${ }^{[7}$ an adaptive observer was proposed to exponentially estimate the state and the unknown parameters under a persistent excitation condition for uniformly observable Multiple-Input-Multiple-Output (MIMO) nonlinear systems. The authors also proposed an adaptive observer for a class of uniformly observable MIMO nonlinear systems with general

\footnotetext{
${ }^{\dagger}$ A preliminary version of this paper has been accepted at the 57th IEEE Conference on Decision and Control 1 !

${ }^{0}$ Abbreviations: Input-to-State-Stable (ISS), Finite-Time Stable (FTS), Multiple-Input Multiple-Output (MIMO), High-Order Slidng-Modes (HOSM), Linear Matrix Inequalities (LMI)
} 
nonlinear parametrization which provides exponential convergence to zero of the adaptive estimation error. $\operatorname{In}{ }^{8}$, based on the concepts of weakly attracting sets and nonuniform convergence, an adaptive observer is proposed for the asymptotic reconstruction of the state and parameter values in a particular class of forward-complete Single-Input Single-Output nonlinear systems. Adaptive observers, using delays are proposed by 9 . The authors provided a method for redesigning adaptive observers for nonlinear systems that increases the computational effort but provides better parameter estimations and some robustness properties. The problem time-varying parameters estimation problem is studied in $\frac{10}{10}$ where a recursive least-squares algorithm is presented to estimate time-varying parameters of missing data systems establishing asymptotic convergence to the real parameters. $\operatorname{In}^{111}$, robust adaptive observer design methodology for a class of uncertain nonlinear systems in presence of time-varying unknown parameters is given. The developed observer achieves asymptotic convergence of state estimation while ensuring boundedness of parameter errors. A novel variable-length sliding window-based least-squares algorithm is developed in $\frac{12}{2}$. Such an algorithm offers a viable alternative to traditional recursive least-squares for on-line time-varying parameter estimation decreasing the computational complexity. In ${ }^{13}$, two identification algorithms are developed in order to identify time-varying parameters in a finite time or prescribed time (fixed-time), the case of noise measurements is analyzed for both algorithms.

In the sliding-mode area, different adaptive observers have been proposed. In ${ }^{14}$ an adaptive sliding-mode observer is designed for the selective catalytic reduction system in diesel-engine after-treatment systems achieving better performance with respect to a Luenberger-like observer. In the fault detection problem, an adaptive sliding-mode observer is proposed by $\frac{15}{15}$ to solve the problem of sensor fault diagnosis in an industrial gas turbine. Such an adaptive observer provides simultaneous fault detection and identification despite the presence of noise. $\mathrm{In}^{16}$ an adaptive-gain second-order sliding-mode observer is developed for estimating the system states and the uncertain parameters. The performance of the proposed observer is illustrated in a fuel cell air-feed system using a hardware-in-loop emulator. An adaptive sliding-mode observer is proposed by ${ }^{17}$ for a class of nonlinear systems with unknown parameters and faults. Based on the main properties of the sliding-mode observers, an asymptotic fault reconstruction is given taking into account that the relative degree of the output, with respect to the fault, is equal to one. $\operatorname{In} \frac{18}{}$, the problem of an adaptive high gain observer for a class of MIMO non uniformly observable systems involving some unknown constant parameters is addressed. Exponential convergence is established under an appropriate persistent excitation property up to the classical high gain state observer design assumptions. It is important to highlight that most of the proposed adaptive observers are based on linear parameter estimation algorithms. Additionally, most of these works do not consider external disturbances and the convergence rate of the state and parameter estimation errors is asymptotic or exponential. In this context, in 19 it was shown that improvements of convergence rate, for the parameter estimation error, cannot be achieved by simply increasing the observer gains but changing the structure of the adaptive observers.

Motivated for the aforementioned issues, in this paper a nonlinear adaptive sliding-mode observer is proposed, based on a nonlinear time-varying parameter identification algorithm, for uncertain nonlinear systems with measurement noise. This nonlinear adaptive observer extends the class of nonlinear systems studied in ${ }^{20}$ considering unknown time-varying parameters, measurement noise, and parameter distribution matrix and nonlinear terms depending on the full state and the input. For the disturbed case, the nonlinear time-varying parameter identification algorithm provides a fixed-time rate of convergence, to a neighborhood of the origin, while the sliding-mode observer ensures ultimate boundness for the state estimation error attenuating the effects of the external disturbances. For the ideal case, i.e. without external disturbances and measurement noise, the adaptive observer provides finite-time convergence to a neighborhood of the origin for the state estimation error and fixed-time convergence to zero for the parameter identification error. The synthesis of the observer is given in terms of LMIs. The convergence proofs are developed based on Lyapunov and Input-to-State Stability (ISS) theory. Some simulation results illustrate the performance of the proposed nonlinear adaptive sliding-mode observer. The preliminary investigations in this direction can be found in ${ }^{\mathbb{1}}$ for the case of finite-time convergence, without proofs and without analysis of the measurement noise influence.

The paper is organized as follows. The problem statement is formulated in Section 2. The preliminaries are discussed in Section 3 The adaptive observer and the main results are presented in Section 4 Simulation results are shown in Section 5 followed by the conclusions in Section 6 Finally, all the proofs are postponed to the Appendix.

Notation: The Euclidean norm of a vector $q \in \mathbb{R}^{n}$ is denoted by $\|q\|$ and define the $p$-norm as $\|q\|_{p}=\left(\sum_{i=1}^{n}\left|q_{i}\right|^{p}\right)^{\frac{1}{p}}$, for $p \geq 1$. For a matrix $Q \in \mathbb{R}^{m \times n}$, denote its smallest singular value $\sigma_{\min }(Q)=\sqrt{\lambda_{\min }\left(Q^{T} Q\right)}$ and $\|Q\|:=\sqrt{\lambda_{\max }\left(Q^{T} Q\right)}=$ $\sigma_{\max }(Q)$, where $\lambda_{\max }$ is the maximum eigenvalue and $\lambda_{\min }$ is the minimum one, $\sigma_{\max }$ is the largest singular value. For a Lebesgue measurable function $u: \mathbb{R}_{\geq 0} \rightarrow \mathbb{R}^{m}$, define the norm $\|u\|_{\left(t_{0}, t_{1}\right)}:=e s s \sup _{t \in\left(t_{0}, t_{1}\right)}\|u(t)\|$, then $\|u\|_{\infty}:=\|u\|_{(0,+\infty)}$ and the set of functions $u$ with the property $\|u\|_{\infty}<+\infty$ is denoted as $\mathcal{L}_{\infty}$. For a matrix $Q: \mathbb{R}_{\geq 0} \rightarrow \mathbb{R}^{m \times n}$, denote $\|Q\|_{\infty}:=\|Q\|_{(0,+\infty)}$. A continuous function $\alpha: \mathbb{R}_{\geq 0} \rightarrow \mathbb{R}_{\geq 0}$ belongs to class $\mathcal{K}$ if it is strictly increasing and $\alpha(0)=0$; it belongs to class $\mathcal{K}_{\infty}$ if it is also unbounded. A continuous function $\beta: \mathbb{R}_{\geq 0} \times \mathbb{R}_{\geq 0} \rightarrow \mathbb{R}_{\geq 0}$ belongs to class $\mathcal{K} \mathcal{L}$ if, for each fixed $s, \beta(r, s) \in \mathcal{K}$ with respect to 
$r$, and for each fixed $r, \beta(r, s)$ is decreasing to zero with respect to $s$. Define the function $\lceil a\rfloor^{\alpha}:=|a|^{\alpha} \operatorname{sign}(a)$, for any $\alpha \in[0,1)$ and any $a \in \mathbb{R}$; and the function $\operatorname{sign}[q]:=\frac{q}{\|q\|}$, for any $q \in \mathbb{R}^{m}$. The term $\nabla V(x) f(x)$ denotes the directional derivative of a continuously differentiable function $V$ with respect to the vector field $f$ evaluated at any point $x$.

\section{2 | PROBLEM STATEMENT}

Consider the following class of uncertain nonlinear systems,

$$
\begin{aligned}
& \dot{x}=A x+\phi(x, u)+G(t, x, u) \theta(t)+D w(t), \\
& y=C x+\varsigma(t),
\end{aligned}
$$

where $x \in \mathbb{R}^{n}$ is the state vector, $y \in \mathbb{R}^{p}$ is the measurable output vector, $u \in \mathbb{R}^{m}$ is the control input vector, $\theta: \mathbb{R}_{\geq 0} \rightarrow \mathbb{R}^{q}$ is a vector of unknown time-varying parameters, $\varsigma: \mathbb{R}_{\geq 0} \rightarrow \mathbb{R}^{p}$ is a vector of measurement noise, and $w: \mathbb{R}_{\geq 0} \rightarrow \mathbb{R}^{l}$ represents the external disturbances. The matrices $A, C$ and $D$ are known, they have corresponding dimensions, and the pair $(A, C)$ is detectable. The functions $\phi: \mathbb{R}^{n} \times \mathbb{R}^{m} \rightarrow \mathbb{R}^{n}$ and $G: \mathbb{R}_{\geq 0} \times \mathbb{R}^{n} \times \mathbb{R}^{m} \rightarrow \mathbb{R}^{n \times q}$ are also known and they ensure uniqueness and existence of solutions for system (1) for all admissible disturbances. It is important to highlight that in $\frac{20}{}$ the functions $\phi(x, u)$ and $G(t, x, u)$ were considered depending only on the measurable output $y$.

The aim of this paper is to provide estimations of the state and parameter vectors, i.e. $x$ and $\theta$, respectively; only using the information of the output $y$ and attenuating as much as possible the effects of the external disturbances $w$ and the noise $\varsigma$.

Let us consider that system (1)-(2) satisfies the following Assumptions.

Assumption 1. $\|x\|_{\infty}<+\infty,\|u\|_{\infty}<+\infty,\|w\|_{\infty}<+\infty$, and $\|G(t, x(t), u(t))\|<+\infty$, for all $t \geq 0$.

Assumption 2. The functions $\phi(u, x)$ and $G(t, x, u)$ are globally Lipschitz with respect to $x$; the parameter vector $\theta$ and its derivative are bounded, i.e. for all $t \geq 0, x, \hat{x} \in \mathbb{R}^{n}$ and $u \in \mathbb{R}^{m}$, the following conditions are satisfied:

$$
\begin{gathered}
\|\phi(x, u)-\phi(\hat{x}, u)\| \leq L_{\phi}\|x-\hat{x}\|, \\
\|G(t, x, u)-G(t, \hat{x}, u)\| \leq L_{G}\|x-\hat{x}\|, \\
\|\theta\|_{\infty} \leq \theta^{+},\|\dot{\theta}\|_{\infty} \leq \bar{\theta},
\end{gathered}
$$

with $L_{\phi}, L_{G}, \theta^{+}, \bar{\theta}>0$ being some known positive constants.

Remark 1. The global Lipschitz condition can be relaxed, under some mild conditions, to a local Lipschitz one. However, the results will be also local.

\section{3 | PRELIMINARIES}

Consider the following nonlinear system,

$$
\dot{x}=f(x, w)
$$

where $x \in \mathbb{R}^{n}$ is the state, $w \in \mathbb{R}^{l}$ is the external disturbance, and $f: \mathbb{R}^{n} \times \mathbb{R}^{l} \rightarrow \mathbb{R}^{n}$ is a locally Lipschitz function. For an initial condition $x_{0} \in \mathbb{R}^{n}$ and an external disturbance $w \in \mathcal{L}_{\infty}$, denote the solution by $x\left(t, x_{0}, \omega\right)$ for any $t \geq 0$ for which the solution exists.

The following stability properties for system $(3)$ are introduced (for more details see $\frac{21,22,20]}{2}$ and ${ }^{23}$ ).

Definition 1. 21. The system (3) is said to be Input-to-State practically Stable (ISpS) if there exist some functions $\beta \in \mathcal{K} \mathcal{L}, \gamma \in \mathcal{K}$ and a constant $\kappa \in \mathbb{R}_{\geq 0}$ such that for any $w \in \mathcal{L}_{\infty}$ and any $x_{0} \in \mathbb{R}^{n}$, the following inequality is satisfied

$$
\left\|x\left(t, x_{0}, w\right)\right\| \leq \beta\left(\left\|x_{0}\right\|, t\right)+\gamma\left(\|w\|_{\infty}\right)+\kappa, \forall t \geq 0 .
$$

If $\kappa=0$, the system (3) is Input-to-State Stable (ISS).

Lemma 1. 24. Let $V: \mathbb{R}^{n} \rightarrow \mathbb{R}_{\geq 0}$ be a smooth function. If there exist some positive constants $\psi_{1}, \psi_{2}, \psi_{3}, \psi_{4}, \kappa>0$, such that

$$
\begin{gathered}
\psi_{1}\|x\|^{2} \leq V(x) \leq \psi_{2}\|x\|^{2}, \\
\nabla V f(x, w) \leq-\psi_{3} V, \forall\|x\| \geq \mu:=\psi_{4}\|w\|_{\infty}+\kappa,
\end{gathered}
$$


then system (3) is ISpS with respect to the input w. Moreover, the following bounds are satisfied:

$$
\begin{aligned}
& \left\|x\left(t, x_{0}, \omega\right)\right\| \leq e^{-\frac{\psi_{3}}{2} t} \sqrt{\frac{\psi_{2}}{\psi_{1}}}\left\|x_{0}\right\|, \forall t \leq T\left(x_{0}\right), \\
& \left\|x\left(t, x_{0}, \omega\right)\right\| \leq \sqrt{\frac{\psi_{2}}{\psi_{1}}} \mu, \forall t>T\left(x_{0}\right),
\end{aligned}
$$

and

$$
T\left(x_{0}\right) \leq \max \left[0, \frac{2\left[\ln \left(\left\|x_{0}\right\|\right)-\ln (\mu)\right]}{\psi_{3}}\right] .
$$

Lemma 2. 20,25. Let $V: \mathbb{R}^{n} \rightarrow \mathbb{R}_{\geq 0}$ be a smooth function. If there exist some positive constants $\psi_{1}, \psi_{2}, \psi_{3}, \psi_{4}, \psi_{5}, \kappa>0$, $\gamma_{1} \in(0,1)$ and $\gamma_{2}>1$ such that

$$
\begin{gathered}
\psi_{1}\|x\|^{2} \leq V(x) \leq \psi_{2}\|x\|^{2}, \\
\nabla V f(x, w) \leq-\psi_{3} V^{\gamma_{1}}-\psi_{4} V^{\gamma_{2}}, \forall\|x\| \geq \mu:=\psi_{5}\|w\|_{\infty}+\kappa,
\end{gathered}
$$

then system (3) is ISpS with respect to the input $w$. Moreover, the following bounds are satisfied:

$$
\left\|x\left(t, x_{0}, \omega\right)\right\| \leq\left\{\begin{array}{l}
\frac{1}{\sqrt{\psi_{1}}}\left[1+\psi_{4}\left(\gamma_{2}-1\right) t\right]^{\frac{1}{2\left(1-\gamma_{2}\right)}}, \forall t \leq T_{1}, \\
\frac{1}{\sqrt{\psi_{1}}}\left[1-\psi_{3}\left(1-\gamma_{1}\right) t\right]^{\frac{1}{2\left(1-\gamma_{1}\right)}}, \forall T_{1}<t \leq T_{1}+T_{2}, \\
\sqrt{\frac{\psi_{2}}{\psi_{1}}} \mu, \forall t>T_{1}+T_{2},
\end{array}\right.
$$

where

$$
T_{1} \leq \frac{1}{\psi_{4}\left(\gamma_{2}-1\right)}, T_{2} \leq \max \left[0, \frac{1-\psi_{1}^{1-\gamma_{1}} \mu^{2\left(1-\gamma_{1}\right)}}{\psi_{3}\left(1-\gamma_{1}\right)}\right] .
$$

Let us consider the following interconnected nonlinear system

$$
\begin{aligned}
& \dot{x}_{1}=f_{1}\left(x_{1}, x_{2}, \omega\right), \\
& \dot{x}_{2}=f_{2}\left(x_{1}, x_{2}, \omega\right),
\end{aligned}
$$

where $x_{i} \in \mathbb{R}^{n_{i}}, w \in \mathbb{R}^{l}$, and $f_{i}: \mathbb{R}^{n_{1}} \times \mathbb{R}^{n_{2}} \times \mathbb{R}^{l} \rightarrow \mathbb{R}^{n_{i}}$ ensures existence of the system solutions at least locally, for $i=1,2$. Suppose that there exist ISpS Lyapunov functions $V_{1}$ and $V_{2}$, for (4) and (5), respectively; and some functions $\psi_{i 1}, \psi_{i 2}, \psi_{i 3} \in \mathcal{K}_{\infty}$, $\gamma_{i}, \chi_{i} \in \mathcal{K}$, and some constants $\kappa_{i} \in \mathbb{R}_{\geq 0}$ such that, for all $x_{i} \in \mathbb{R}^{n_{i}}$ and any $w \in \mathcal{L}_{\infty}$, the following hold:

$$
\begin{gathered}
\psi_{i 1}\left(\left\|x_{i}\right\|\right) \leq V_{i}\left(x_{i}\right) \leq \psi_{i 2}\left(\left\|x_{i}\right\|\right), i=1,2, \\
V_{1}\left(x_{1}\right) \geq \max \left[\chi_{1}\left(V_{2}\left(x_{2}\right)\right), \gamma_{1}(\|w\|)+\kappa_{1}\right] \Rightarrow \nabla V_{1}\left(x_{1}\right) f_{1}\left(x_{1}, x_{2}, \omega\right) \leq-\psi_{13}\left(V_{1}\left(x_{1}\right)\right), \\
V_{2}\left(x_{2}\right) \geq \max \left[\chi_{2}\left(V_{1}\left(x_{1}\right)\right), \gamma_{2}(\|w\|)+\kappa_{2}\right] \Rightarrow \nabla V_{2}\left(x_{2}\right) f_{2}\left(x_{1}, x_{2}, \omega\right) \leq-\psi_{23}\left(V_{2}\left(x_{2}\right)\right) .
\end{gathered}
$$

Thus, the following nonlinear small-gain result is introduced, in terms of ISpS Lyapunov properties, for the interconnected system (4)-(5).

Theorem 1. 22. Suppose that the interconnected system (4)-(5) has ISpS Lyapunov functions $V_{1}$ and $V_{2}$ satisfying the conditions (6)-(8). Then, the system (4)-(5) is ISpS if

$$
\chi_{1} \circ \chi_{2}(r)<r, \forall r>\kappa_{0},
$$

for some $\kappa_{0} \in \mathbb{R}_{\geq 0}$. System (4)-(5) is ISS if $\kappa_{0}=\kappa_{1}=\kappa_{2}=0$.

\section{4 | ADAPTIVE SLIDING-MODE OBSERVER}

Let us introduce the following adaptive observer

$$
\begin{aligned}
\dot{\Omega} & =(A-L C) \Omega+G(t, \hat{x}, u), \\
\dot{\hat{\theta}} & =\Gamma \Omega^{T} C^{T}\left[\lceil y-C \hat{x}\rfloor^{\alpha}+\lceil y-C \hat{x}\rfloor^{\beta}\right], \\
\dot{\hat{x}} & =A \hat{x}+\phi(\hat{x}, u)+G(t, \hat{x}, u) \hat{\theta}+L(y-C \hat{x})+k D \operatorname{sign}[F(y-C \hat{x})]+\Omega \dot{\hat{\theta}},
\end{aligned}
$$


where $\hat{\theta} \in \mathbb{R}^{q}$ and $\hat{x} \in \mathbb{R}^{n}$ are the estimations for $\theta$ and $x$, respectively; and $\Omega \in \mathbb{R}^{n \times q}$ is an auxiliary variable. If the signal $G$ is persistently exciting (PE) (see, e.g..$^{[2}$ and ${ }^{3}$ ), then, due to the stable filtering property of the variable $\Omega$ and the detectability of the pair $(A, C)$, the variable $C \Omega$ is also PE. Note that the functions $[y-C \hat{x}\rfloor^{\alpha}$ and $[y-C \hat{x}\rfloor^{\beta}$, with $\alpha \in[0,1)$ and $\beta>1$, in (10) are understood in the component-wise sense. The observer matrix gain $L \in \mathbb{R}^{n \times p}$ has to be selected such that $(A-L C)$ is Hurwitz, $0<\Gamma=\Gamma^{T} \in \mathbb{R}^{q \times q}$, while $F \in \mathbb{R}^{l \times p}, k \geq 0$ are designed later. Since (11) is discontinuous, its solutions are understood in the Filippov sense (for more details, see ${ }^{26}$ and ${ }^{27}$ ), i.e. the solutions are defined as absolutely continuous functions of time satisfying the corresponding Filippov inclusion almost everywhere, and they always exist and have most of the wellknown differential equations standard properties except the uniqueness. The ISS concepts have been extended to these classes of systems (see $e . g .{ }^{28}$ ).

Let us introduce the following hypothesis:

Assumption 3. Let $0<\rho_{\min } \leq \sigma_{\min }(C \Omega(t))$ for all $t \geq 0$ and $\|C \Omega\|_{\infty} \leq \rho_{\max }<+\infty$.

The existence of $\varrho_{\max }$ is guaranteed by Assumption 1 and the fact that matrix $(A-L C)$ is Hurwitz; the existence of $\varrho_{\min }$ follows, for example, if $C$ has full row rank with $p \geq q$; then, $\Omega(0)$ can always be selected such that $0<\rho_{\min } \leq \sigma_{\min }(C \Omega(0)$ ); and hence, such a property is preserved, for all $t>0$, by continuity and providing that $G$ is PE (for more details, see ${ }^{29}$ ). The values of $\varrho_{\max }$ and $\varrho_{\min }$ can be evaluated numerically during experiments.

\section{1 | Nonlinear Parameter Identification Algorithm}

Define $\tilde{\theta}:=\hat{\theta}-\theta$ and $\delta:=\tilde{x}+\Omega \tilde{\theta}$ with $\tilde{x}:=x-\hat{x}$. Then, the error dynamics are given by:

$$
\begin{aligned}
& \dot{\tilde{\theta}}=-\Gamma \Omega^{T} C^{T}\left[\lceil C \Omega \tilde{\theta}-C \delta-\varsigma]^{\alpha}+\lceil C \Omega \tilde{\theta}-C \delta-\varsigma]^{\beta}\right]-\dot{\theta}, \\
& \dot{\delta}=(A-L C) \delta+D(w-k \operatorname{sign}[F(y-C \hat{x})])+\Delta_{\phi}(x, \hat{x}, u)+\Delta_{G}(t, x, \hat{x}, u) \theta-\Omega \dot{\theta}+L \varsigma,
\end{aligned}
$$

where $\Delta_{\phi}(x, \hat{x}, u):=\phi(x, u)-\phi(\hat{x}, u)$ and $\Delta_{G}(t, x, \hat{x}, u):=G(t, x, u)-G(t, \hat{x}, u)$. The ISpS convergence properties of the error dynamics (12) with respect to the inputs $\delta$ and $\varsigma$, for $\alpha \in(0,1)$ and $\alpha=0$, with $\beta>1$, are given by the following lemmas.

Lemma 3. Let Assumption 3 be satisfied. Then, the error dynamics (12), with $\alpha \in(0,1), \beta>1$ and $\Gamma=\Gamma^{T}>0$, is ISpS with respect to $\delta$ and $\varsigma$, and the trajectories satisfy:

$$
\begin{aligned}
& \|\tilde{\theta}\| \leq \frac{1}{\sqrt{c_{1}}}\left(1+\frac{p^{\frac{1-\beta}{2(\beta+1)}} k_{12}\left(1-\varphi_{2}\right)(\beta-1) \rho_{\min }^{\beta+1} c_{2}^{\frac{\beta+1}{2}}}{2} t\right)^{\frac{1}{1-\beta}}, \forall t \leq T_{\tilde{\theta}_{1}}, \\
& \|\tilde{\theta}\| \leq \frac{1}{\sqrt{c_{1}}}\left(1-\frac{k_{11}\left(1-\varphi_{1}\right)(1-\alpha) \rho_{\min }^{\alpha+1} c_{2}^{\frac{\alpha+1}{2}}}{2} t\right)^{\frac{1}{1-\alpha}}, \forall T_{\tilde{\theta}_{1}}<t \leq T_{\tilde{\theta}_{1}}+T_{\tilde{\theta}_{2}}, \\
& \|\tilde{\theta}\| \leq \sqrt{\frac{c_{1}}{c_{2}}} \mu_{\tilde{\theta}_{1}}, \forall t>T_{\tilde{\theta}_{1}}+T_{\tilde{\theta}_{2}},
\end{aligned}
$$

where $c_{1}:=2 \lambda_{\max }(\Gamma), c_{2}:=2 \lambda_{\min }(\Gamma), k_{11}, k_{12} \in(0,1)$ and

$$
\mu_{\tilde{\theta}_{1}}=\max \left[\frac{\left(2 p k_{21}\right)^{\frac{1}{\alpha+1}}}{\left(\varphi_{1} k_{11}\right)^{\frac{1}{\alpha+1}} \varrho_{\min }}\left(\|C\|\|\mid \delta\|_{\infty}+\|\varsigma\|_{\infty}\right)+\kappa_{\tilde{\theta} 1}, \frac{\left(2 k_{22}\right)^{\frac{1}{\beta+1}}}{\left(p^{\frac{1-\beta}{2(\beta+1}} \varphi k_{12}\right)^{\frac{1}{\beta+1}} \varrho_{\min }}\left(\|C\|\|\mid \delta\|_{\infty}+\|\varsigma\|_{\infty}\right)\right] \text {, }
$$

with

$$
\kappa_{\tilde{\theta} 1}=\left(\frac{4 c_{2}^{-1} \bar{\theta}}{\varphi k_{11} \rho_{\min }^{\alpha+1}}\right)^{\frac{1}{\alpha}}, T_{\tilde{\theta}_{1}} \leq \frac{2}{p^{\frac{1-\beta}{2(\beta+1)}} k_{12}\left(1-\varphi_{2}\right)(\beta-1) \rho_{\min }^{\beta+1} c_{2}^{\frac{\beta+1}{2}}}, T_{\tilde{\theta}_{2}} \leq \max \left[0, \frac{2-2 c_{1}^{\frac{\alpha-1}{2}} \mu_{\tilde{\theta}_{1}}^{1-\alpha}}{k_{11}\left(1-\varphi_{1}\right)(1-\alpha) \varrho_{\min }^{\alpha+1} c_{2}^{\frac{\alpha+1}{2}}}\right],
$$

for any $\varphi_{1}, \varphi_{2} \in(0,1), k_{21}=\max \left(k_{11}+1, k_{11} /\left(1-k_{11}^{\frac{1}{\alpha}}\right)^{\alpha}\right)$ and $k_{22}=\max \left(k_{12}+1, k_{12} /\left(1-k_{12}^{\frac{1}{\beta}}\right)^{\beta}\right.$. 
Lemma 4. Let Assumption 3 be satisfied. Then, the error dynamics (12), with $\alpha=0, \beta>1$ and $\lambda_{\min }(\Gamma) \geq 2 \bar{\theta} / \varphi k_{11} \varrho_{\min }$, is ISS with respect to $\delta, \varsigma$ and the trajectories satisfy:

$$
\begin{aligned}
& \|\tilde{\theta}\| \leq \frac{1}{\sqrt{c_{1}}}\left(1+\frac{\frac{1-\beta}{2(\beta+1)}^{\frac{1-\beta}{12}} k_{12}\left(1-\varphi_{2}\right)(\beta-1) \rho_{\min }^{\beta+1} c_{2}^{\frac{\beta+1}{2}}}{2} t\right)^{\frac{1}{1-\beta}}, \forall t \leq T_{\tilde{\theta}_{3}} \\
& \|\tilde{\theta}\| \leq \frac{1}{\sqrt{c_{1}}}\left(1-k_{11}\left(1-\varphi_{1}\right) \rho_{\min } c_{2}^{\frac{1}{2}} t\right), \forall T_{\tilde{\theta}_{3}}<t \leq T_{\tilde{\theta}_{3}}+T_{\tilde{\theta}_{4}}, \\
& \|\tilde{\theta}\| \leq \sqrt{\frac{c_{1}}{c_{2}}} \mu_{\tilde{\theta}_{2}}, \forall t>T_{\tilde{\theta}_{3}}+T_{\tilde{\theta}_{4}},
\end{aligned}
$$

where $\tilde{\theta}_{0}:=\tilde{\theta}(0), c_{1}:=2 \lambda_{\max }(\Gamma), c_{2}:=2 \lambda_{\min }(\Gamma), k_{11}, k_{12} \in(0,1)$ and

$$
\begin{gathered}
\mu_{\tilde{\theta}_{2}}=\max \left[\frac{2 \sqrt{p} k_{21}}{\varphi_{1} k_{11} \varrho_{\min }}, \frac{\left(2 k_{22}\right)^{\frac{1}{\beta+1}}}{\left(p^{\frac{1-\beta}{2(\beta+1)}} \varphi k_{12}\right)^{\frac{1}{\beta+1}} \varrho_{\min }}\right]\left(\|C\|\|\delta\|_{\infty}+\|\varsigma\|_{\infty}\right), \\
T_{\tilde{\theta}_{3}} \leq \frac{2}{p^{\frac{1-\beta}{2(\beta+1)}} k_{12}\left(1-\varphi_{2}\right)(\beta-1) \varrho_{\min }^{\beta+1} c_{2}^{\frac{\beta+1}{2}}}, T_{\tilde{\theta}_{4}} \leq \max \left[0, \frac{2-2 c_{1}^{-\frac{1}{2}} \mu_{\tilde{\theta}_{2}}}{k_{11}\left(1-\varphi_{1}\right) \varrho_{\min } c_{2}^{\frac{1}{2}}}\right],
\end{gathered}
$$

for any $\varphi_{1}, \varphi_{2} \in(0,1), k_{21} \geq k_{11}+1$ and $k_{22}=\max \left(k_{12}+1, k_{12} /\left(1-k_{12}^{\frac{1}{\beta}}\right)^{\beta}\right.$.

The results of lemmas 3 and 4 establish that the trajectories of the error dynamics (12), i.e. $\tilde{\theta}(t)$, enter into the bound $\sqrt{c_{1} / c_{2}} \mu_{\tilde{\theta}_{1}}$ or $\sqrt{c_{1} / c_{2}} \mu_{\tilde{\theta}_{2}}$ in a fixed time for any $\alpha \in[0,1)$ and $\beta>1$. It is clear that the size of such a bound depends on $\alpha$ and $\beta$. In the same vein, the following lemma illustrates the ISpS convergence properties of the error dynamics (13) with respect to the inputs $\tilde{\theta}, \varsigma$ and $w$.

Lemma 5. Let assumptions 1 and 2 hold. Assume that $k \geq\|w\|_{\infty}+\gamma$, with $\gamma>0$, and that there exist $0<P=P^{T} \in \mathbb{R}^{n \times n}$, $F \in \mathbb{R}^{l \times p}, L \in \mathbb{R}^{n \times p}$ and some $\vartheta, \varepsilon, \sigma>0$ such that the following matrix inequalities

$$
\begin{aligned}
& P D \leq C^{T} F^{T}, \\
& \left(\begin{array}{cccc}
(A-L C)^{T} P+P(A-L C)+\eta_{1} P+\sigma C^{T} C & \frac{\vartheta}{2} I_{n} & P & P \\
\star & -\Lambda^{-1} & 0 & 0 \\
\star & \star & -\Lambda & 0 \\
\star & \star & \star & -\frac{\varepsilon}{\eta_{2}^{2}} I_{n}
\end{array}\right) \leq 0,
\end{aligned}
$$

are feasible for $0<\Lambda^{T}=\Lambda \in \mathbb{R}^{n \times n}, \eta_{1}:=2\left(L_{\phi}+L_{G} \theta^{+}\right)$and $\eta_{2}:=\eta_{1} \varrho_{\max }\|C\|^{-1}$. Then, the error dynamics $(13)$ is ISpS with respect to the inputs $\tilde{\theta}, \varsigma$ and $w$. Furthermore, the trajectories satisfy:

$$
\begin{aligned}
& \|\delta(t)\| \leq e^{-\frac{(1-\rho) \alpha_{\delta}}{2 r_{2}} t} \sqrt{\frac{r_{2}}{r_{1}}}\left\|\delta_{0}\right\|, \forall t \leq T_{\delta_{0}}, \\
& \|\delta(t)\| \leq \sqrt{\frac{r_{2}}{r_{1}}} \mu_{\delta}, \forall t>T_{\delta_{0}},
\end{aligned}
$$

where $\delta_{0}:=\delta(0), \alpha_{\delta}:=\left(\vartheta r_{1}+\sigma\|C\|^{2}\right) / 2, r_{1}=\lambda_{\min }(P), r_{2}=\lambda_{\max }(P)$ and

$$
\begin{gathered}
\mu_{\delta}=\sqrt{\frac{2 \varepsilon+\sigma \varrho_{\max }^{2}}{2 \rho \alpha_{\delta}}}\|\tilde{\theta}\|_{\infty}+\sqrt{\frac{2 r_{2}^{2}}{\vartheta r_{1} \rho \alpha_{\delta}}}\|\varsigma\|\|L\|+\sqrt{\frac{8\|F\|^{2}}{\rho \alpha_{\delta} \sigma}}\|w\|_{\infty}+\kappa_{\delta}, \kappa_{\delta}=\sqrt{\frac{2 r_{2}^{2}}{\vartheta r_{1} \rho \alpha_{\delta}}}\left(\bar{\theta} \rho_{\max }\|C\|^{-1}\right), \\
T_{\delta} \leq \max \left(0, \frac{2 r_{2}\left[\ln \left(\left\|\delta_{0}\right\|\right)-\ln \left(\mu_{\delta}\right)\right]}{(1-\rho) \alpha_{\delta}}\right),
\end{gathered}
$$

for any $\delta_{0} \in \mathbb{R}^{n}$ and $\rho \in(0,1)$.

It is clear that the size of $\mu_{\delta}$ could be minimized in order to attenuate the effects of $w$ and $\varsigma$. 


\section{2 | Convergence of the Adaptive Observer}

In order to show the convergence properties of the adaptive observer (9)-(11), the statements provided by lemmas $3,4,5$ and Theorem 1 are applied. The following results show that the interconnected error system (12)-(13) is ISpS with respect to the external disturbances $w$ and measurement noise $\varsigma$ for any $\alpha \in(0,1)$ and $\alpha=0$, respectively; and $\beta>1$.

Theorem 2. Let assumptions 1,2 and 3 hold. Assume that there exist $0<P=P^{T} \in \mathbb{R}^{n \times n}, F \in \mathbb{R}^{l \times p}, L \in \mathbb{R}^{n \times p}$ and some $\vartheta, \varepsilon, \sigma>0$ such that matrix inequalities (20) are feasible for $0<\Lambda^{T}=\Lambda \in \mathbb{R}^{n \times n}, \eta_{1}=2\left(L_{\phi}+L_{G} \theta^{+}\right)$and $\eta_{2}=\eta_{1} \varrho_{\max }\|C\|^{-1}$. Then, for any $\Gamma=\Gamma^{T}>0, \alpha \in(0,1)$, with $\beta>1$, the interconnected error system (12)-(13) is ISpS with respect to the inputs $w$ and $\varsigma$.

Theorem 3. Let assumptions 1,2 and 3 hold. Assume that there exist $0<P=P^{T} \in \mathbb{R}^{n \times n}, F \in \mathbb{R}^{l \times p}, L \in \mathbb{R}^{n \times p}$ and some $\vartheta, \varepsilon, \sigma>0$ such that matrix inequalities (20) are feasible for $0<\Lambda^{T}=\Lambda \in \mathbb{R}^{n \times n}, \eta_{1}=2\left(L_{\phi}+L_{G} \theta^{+}\right)$and $\eta_{2}=\eta_{1} \varrho_{\max }\|C\|^{-1}$. Then, for any $\Gamma=\Gamma^{T}>0, \alpha=0$, with $\beta>1$, the interconnected error system (12)-(13) is ISpS with respect to the inputs $w$ and $\varsigma$.

Theorems 2 and 3 imply that the estimation error $e:=x-\hat{x}=\delta+\Omega \tilde{\theta}$ is also ISpS with respect to $w$ and $\varsigma$, for any $\alpha \in[0,1)$ and $\beta>1$, since

$$
\|e(t)\| \leq(1+\|\Omega\|)\|(\tilde{\theta}(t) \delta(t))\|,
$$

for all $t \geq 0$.

\section{REMARKS}

1. In the ideal case, i.e. $w=\varsigma \equiv 0$ and $k=0$, the estimation of $\hat{\theta}$ converges in a fixed time to the real value for $\alpha=0$ and any $\beta>1$. Meanwhile the estimation $\hat{x}$ converges to a neighborhood close to the real value. For $\alpha \in(0,1)$ and $\beta>1$, the estimations of $\hat{\theta}$ and $\hat{x}$ converge to a neighborhood of the origin. Moreover, if unknown constant parameters are taken into account; then, the state estimation and the parameter identification errors will converge to zero exponentially and in a fixed time, respectively. However, for constant parameters, the uniform injectivity of $C \Omega(t)$ is just sufficient and stronger than the necessary PE condition.

2. For the noise-free case, i.e. $w \not \equiv 0$ and $\varsigma=0$, the effect of the external disturbance $w$ is completely attenuated if one fixes $k \geq\|w\|_{\infty}+\gamma$, with $\gamma>0$. This can be shown by taking $V_{e}=e^{T} P e$ which implies that

$$
\dot{V}_{e} \leq e^{T}\left[(A-L C)^{T} P+P(A-L C)\right] e+2 e^{T} P\left(\Delta_{\phi}+\Delta_{G} \theta\right)-2 e^{T} P[G \tilde{\theta}+\Omega \dot{\hat{\theta}}+L \varsigma]+2 e^{T} C^{T} F^{T}[w-k \operatorname{sign}[F(C e)]],
$$

and selecting $k \geq\|w\|_{\infty}+\gamma$, with $\gamma>0$, it follows that

$$
\dot{V}_{e} \leq e^{T}\left[(A-L C)^{T} P+P(A-L C)\right] e+2 e^{T} P\left(\Delta_{\phi}+\Delta_{G} \theta\right)-2 e^{T} P[G \tilde{\theta}+\Omega \dot{\hat{\theta}}+L \varsigma] .
$$

3. Note that condition $P D \leq C^{T} F^{T}$ introduces some structural restrictions. Particularly, one has that the triple $(A, D, C)$ must not have invariant zeros, and the relative degree of the output $F y$ with respect to the input $w$ must be equal to one.

4. To find a solution of the matrix inequality [20], one can rewrite it as follows:

$$
(A-L C)^{T} P+P(A-L C)+\vartheta P+\sigma C^{T} C+\eta_{1} P+\epsilon^{-1} \eta_{2}^{2} P^{2} \leq 0 .
$$

Using the fact that $X Y^{T}+Y X^{T} \leq X \Lambda^{-1} X^{T}+Y \Lambda Y^{T}$ holds for every $X \in \mathbb{R}^{k \times n}, Y \in \mathbb{R}^{k \times n}$, and $0<\Lambda=\Lambda^{T} \in \mathbb{R}^{n \times n}$ and applying Schur's complement ${ }^{30}$, it follows that

$$
\left(\begin{array}{cccc}
A^{T} P+P A-C^{T} Y^{T}-Y C+\eta_{1} P+\sigma C^{T} C & \frac{\vartheta}{2} I_{n} & P & P \\
\star & -\Lambda^{-1} & 0 & 0 \\
\star & \star & -\Lambda^{-1} & 0 \\
\star & \star & \star & -\frac{\epsilon}{\eta_{2}^{2}} I_{n}
\end{array}\right) \leq 0,
$$

Note that 23 is an LMI with respect to matrices $P$ and $Y$ and parameters $\vartheta, \varepsilon$ and $\sigma$. Therefore, it is possible to compute the design matrix as $L=P^{-1} Y$.

5. The feasibility of $(20)$ can be ensured, for small enough $\eta_{1}$ and $\eta_{2}$, due to the fact that the pair $(A, C)$ is detectable. 
6. The parameter $\mu_{\delta}$ can be rewritten as follows

$$
\mu_{\delta}=\sqrt{\frac{2 \varepsilon+\sigma \varrho_{\max }^{2}}{\rho\left(\vartheta \lambda_{\min }(P)+\sigma\|C\|^{2}\right)}}\|\tilde{\theta}\|_{\infty}+\sqrt{\frac{8\|F\|^{2}}{\rho \sigma\left(\vartheta \lambda_{\min }(P)+\sigma\|C\|^{2}\right)}}\|w\|_{\infty}+\sqrt{\frac{2 \lambda_{\max }(P)^{2}\left(\bar{\theta} \varrho_{\max }\|C\|^{-1}+\bar{\zeta}\|L\|\right)^{2}}{\rho \vartheta \lambda_{\min }(P)\left(\vartheta \lambda_{\min }(P)+\sigma\|C\|^{2}\right)}} .
$$

Then, in order to minimize the size of $\mu_{\delta}$, it is possible to propose the following optimization problem.

Optimization problem. The minimization of $\mu_{\delta}$, for a fixed $\rho \in(0,1)$ and a matrix $F$ satisfying [20, is equivalent to maximize the size of $P, \vartheta$ and $\sigma$. One simple way is to maximize the trace of $P$ and the values of $\vartheta$ and $\sigma$, i.e.

$$
\begin{aligned}
& \operatorname{tr}(P), \vartheta, \sigma \rightarrow \max _{P, F, Y, \vartheta, \varepsilon, \sigma} \\
& \text { subject to (18) and (21). }
\end{aligned}
$$

7. From the structure of $\mu_{\tilde{\theta}_{1}}$ and $\mu_{\tilde{\theta}_{2}}$, it is clear that the minimum parameter estimation error is obtained when $\alpha=0$, i.e. $\mu_{\tilde{\theta}_{2}} \leq \mu_{\tilde{\theta}_{1}}$. This shows that, with $\alpha=0$, one obtains the best performance in terms of the error convergence to a neighbourhood.

8. Note that $\mu_{\tilde{\theta}_{2}}$ cannot be minimized by any observer matrix gain.

\section{5 | SIMULATION RESULTS}

The corresponding simulations have been done in MATLAB Simulink with the Euler explicit discretization method and sampling time equal to 0.001, while the solution to the given LMIs were obtained by means of SeDuMi solver among YALMIP in MATLAB.

Let us consider a robotic system depicted by Fig. 1 Thus, the robot manipulator dynamics in state-space form (1)-(2) can be

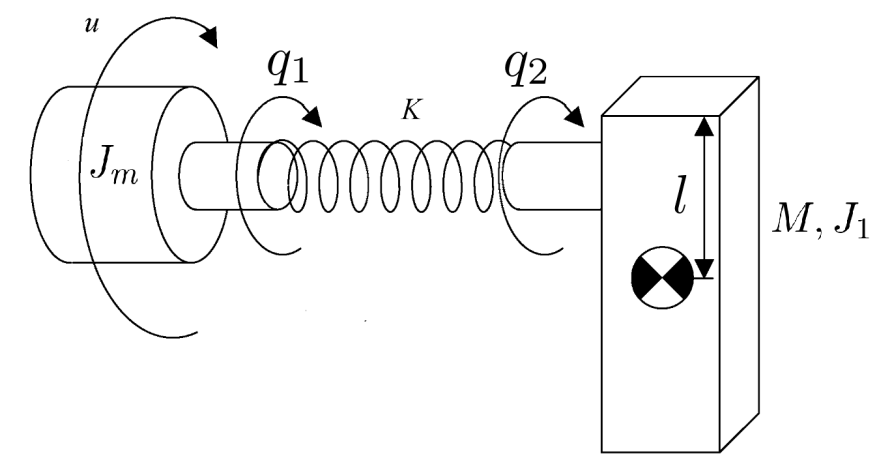

FIGURE 1 Single-link Flexible-joint Robot Manipulator

written as follows $\frac{31}{11}$

$$
\begin{aligned}
& \dot{x}=\left(\begin{array}{cccc}
0 & 1 & 0 & 0 \\
\frac{-k_{s}}{J_{1}} & 0 & \frac{k_{s}}{J_{1}} & 0 \\
0 & 0 & 0 & 1 \\
\frac{k_{s}}{J_{m}} & 0 & \frac{-k_{s}}{J_{m}} & 0
\end{array}\right)+\left(\begin{array}{c}
0 \\
\frac{-M g l}{J_{1}} \sin \left(x_{1}\right) \\
0 \\
\frac{1}{J_{m}} u
\end{array}\right)+\left(\begin{array}{c}
0 \\
0 \\
0 \\
\frac{-x_{4}}{J_{m}}
\end{array}\right) \theta(t)+\left(\begin{array}{l}
0 \\
1 \\
0 \\
0
\end{array}\right) \omega(t), \\
& y=\left(\begin{array}{llll}
1 & 0 & 0 & 0 \\
0 & 1 & 0 & 0
\end{array}\right) x+\varsigma(t),
\end{aligned}
$$

where $x=\left(x_{1}, x_{2}, x_{3}, x_{4}\right)^{T}=\left(q_{1}, q_{3}, q_{2}, q_{4}\right)^{T}, x_{1} \in \mathbb{R}$ and $x_{3} \in \mathbb{R}$ are the angular positions of the link and motor and $x_{2}$ and $x_{4}$ are the velocities of the link and motor, respectively; $J_{1} \in \mathbb{R}_{\geq 0}$ and $J_{m} \in \mathbb{R}_{\geq 0}$ denote the inertia of the link and the motor, $M \in \mathbb{R}_{\geq 0}$ is the mass of the link, $g \in \mathbb{R}_{\geq 0}$ is the gravitational acceleration, $l \in \mathbb{R}_{\geq 0}$ represents the length of the link, $k_{s} \in \mathbb{R}_{\geq 0}$ is 
the stiffness of the spring and $\theta(t)=\sin (0.1 t)+1.5\left[\mathrm{Kgm}^{2} / \mathrm{s}\right]$ is the friction coefficient of the motor which is the unknown timevarying parameter to estimate also $\varsigma$ represents band limited white noise in the output. The numerical values of the parameters are $J_{1}=0.8\left[\mathrm{Kgm}^{2}\right], J_{m}=1\left[\mathrm{Kgm}^{2}\right], g=9.8\left[\mathrm{~m} / \mathrm{s}^{2}\right], l=0.6[\mathrm{~m}], k_{s}=20[\mathrm{Nm} / \mathrm{rad}]$ and $\varsigma$ with a power noise of $1 \times 10^{-6}$. It can be shown that such a system satisfies assumptions 1,2 and 3

Consider the initial conditions $x(0)=(-2,-2,-1.5,2)^{T}$ while the observer initial conditions $\Omega(0)=[0.1,0.1,0.1,0.1]^{T}$, $\hat{\theta}(0)=0$ and $\hat{x}(0)=0$. For simulation purposes, the input is given as $u(t)=\sin (0.2 t)+0.5 \sin (3 t)+2 \cos (50 t)$ while the disturbance term is taken as $w(t)=0.3 \cos (2 t)+0.1$.

Fixing $k=1$ and $\Lambda=I_{4}$. The following feasible solution is found with $L_{\phi}=7, L_{G}=2,\|\Omega\|_{\infty}=0.834$ and

$$
\begin{gathered}
P=\left(\begin{array}{cccc}
1133.3 & -3332.2 & 14.8 & 66.9 \\
-3332.2 & 2192.6 & -87.24 & -24.3 \\
14.8 & -87.24 & 1168.1 & -5.13 \\
66.9 & -24.3 & -5.13 & 27.1
\end{array}\right), \\
L=\left(\begin{array}{cc}
30.2427 & 0.4684 \\
-25.0825 & 93.6232 \\
-0.3414 & 127.9623 \\
18.4329 & 1412.0191
\end{array}\right), F=\left(\begin{array}{l}
293.5014 \\
322.8523
\end{array}\right)^{T},
\end{gathered}
$$

with $\vartheta=2.08 \times 10^{13}, \varepsilon=0.8358$ and $\sigma=8.65 \times 10^{12}$. The simulation results for different values of $\alpha \in[0,1)$ with the same value of $\beta=1.3$, and fixing $\Gamma=3 \times 10^{5}$, that illustrate the statements of Theorem 2 and 3 are depicted by Figs. 2, 3 and 4 . It is clear that due to the disturbances and measurement noise, the corresponding estimations converge to a neighbourhood of the real value but faster than the linear algorithm, i.e. when $\alpha=\beta=1$. In order to better illustrate that the size of the convergence

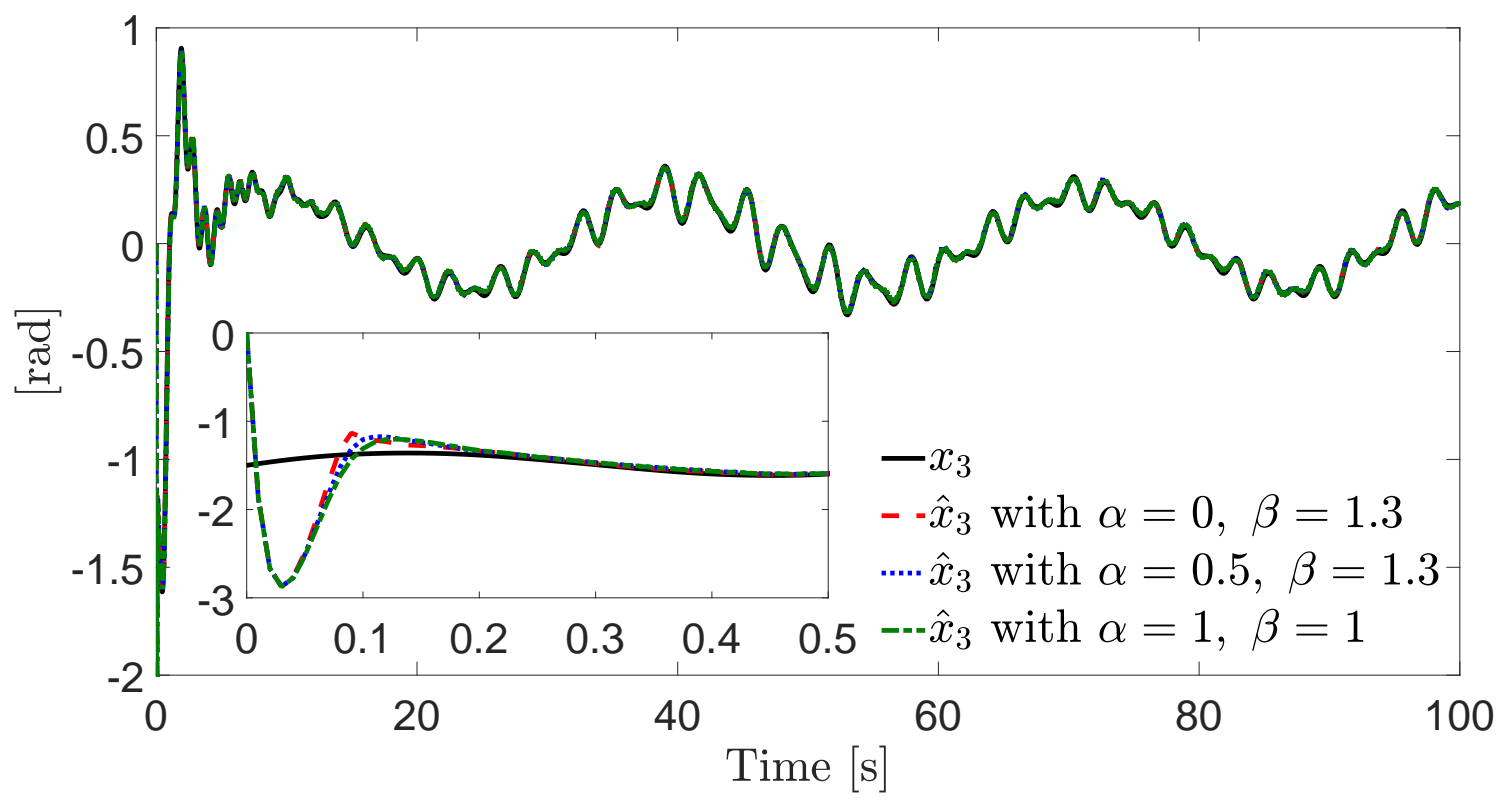

FIGURE 2 Estimation of $x_{3}$

neighbourhood can be improved with respect to the selection of $\alpha$, the following error index is proposed:

$$
e_{R M S}(t)=\left(\frac{1}{\Delta T} \int_{t-\Delta t}^{t}\|\tilde{\theta}(\tau), e(\tau)\|^{2} d \tau\right)^{\frac{1}{2}},
$$

where $\Delta T=2$ is a time window width in which the corresponding sinal is evaluated. The results are depicted by Fig. 5 also an average of the index is shown in Table 1 It is clear that for $\alpha=0$ one obtains the best performance. 


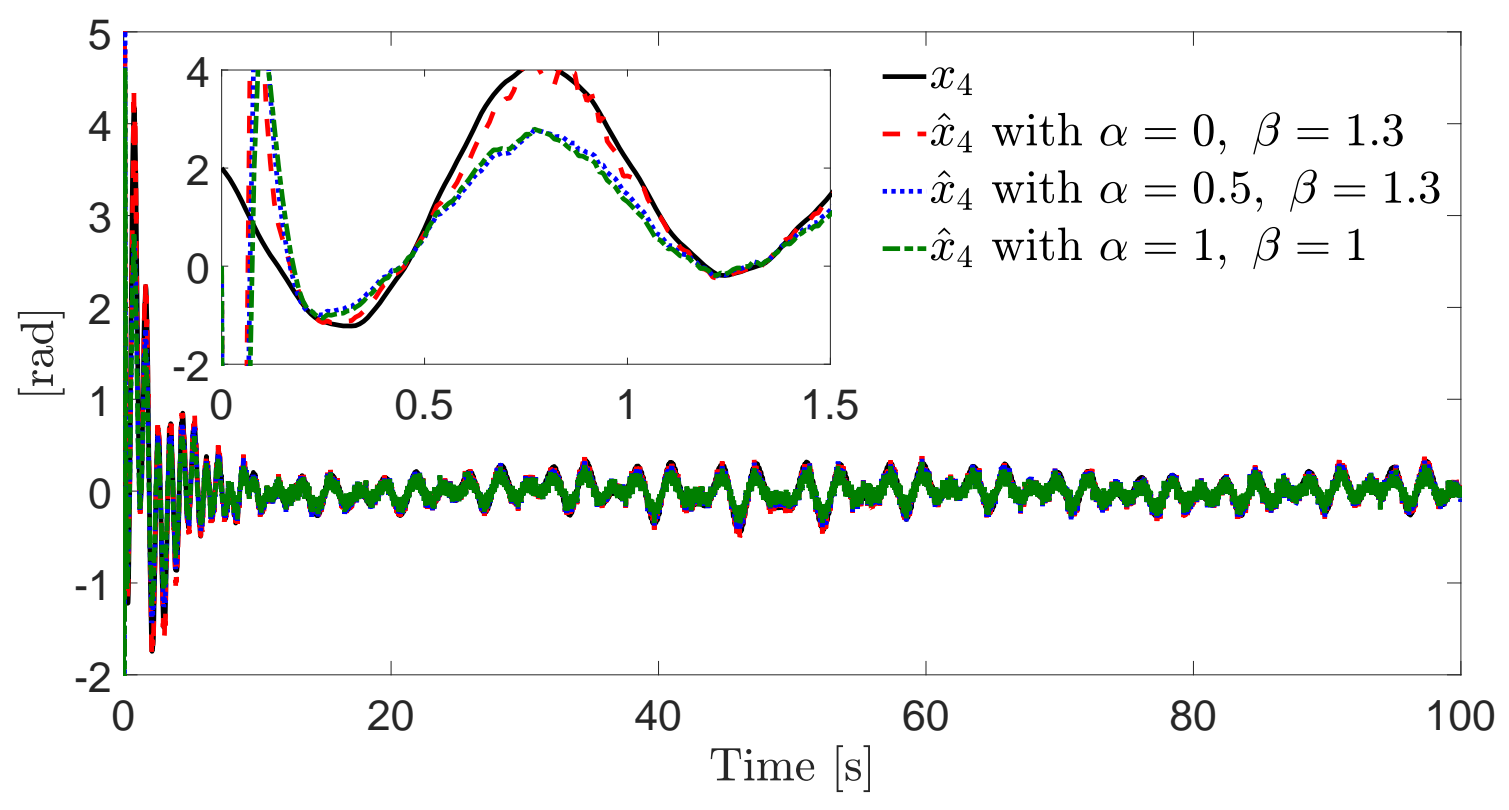

FIGURE 3 Estimation of $x_{4}$

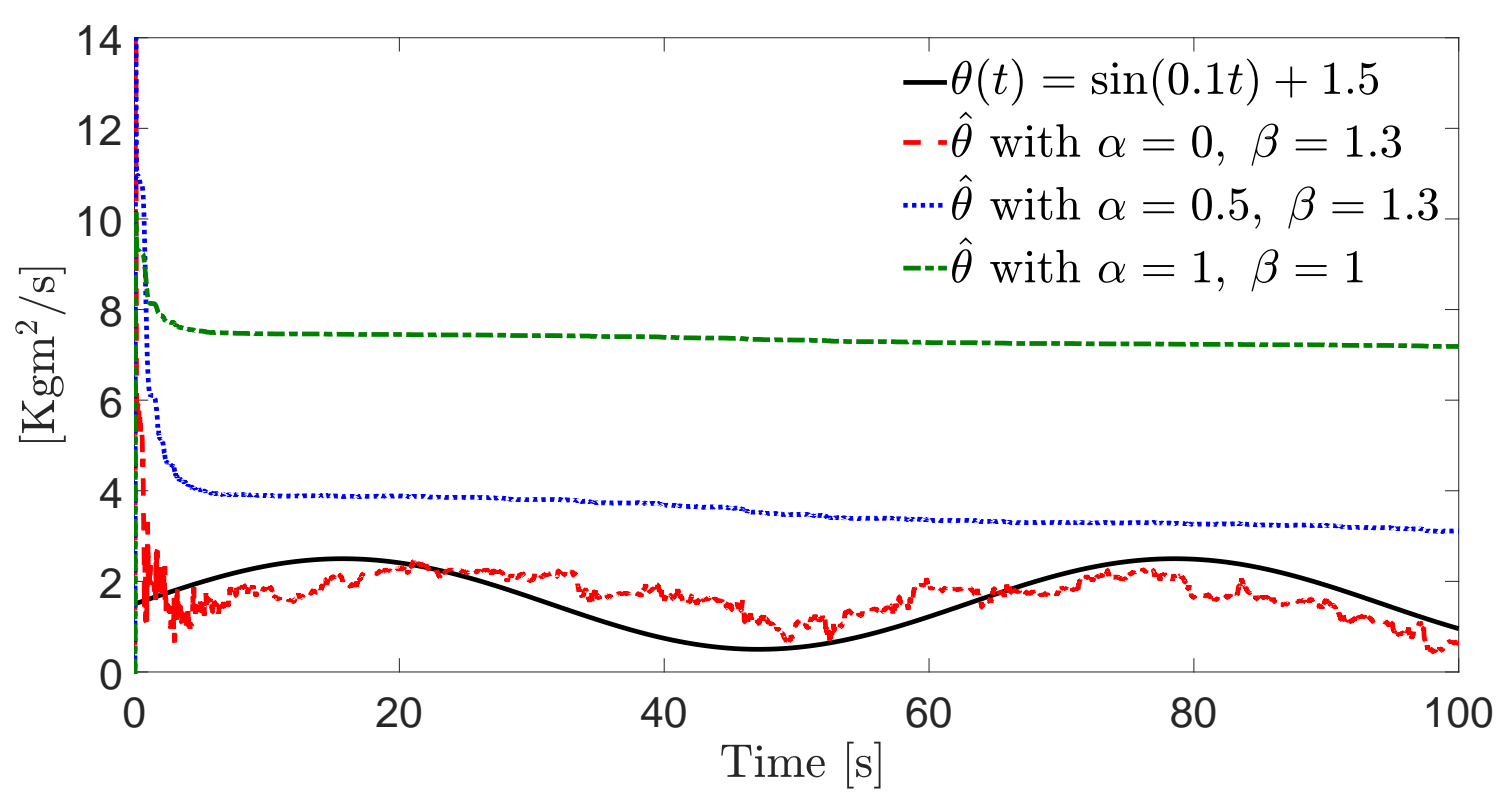

FIGURE 4 Estimation of $\theta(t)$

\begin{tabular}{|c|c|}
\hline $\boldsymbol{\alpha}$ & Error index \\
\hline 0 & 0.6174 \\
\hline 0.5 & 1.9947 \\
\hline 1 & 5.6703 \\
\hline
\end{tabular}

TABLE 1 Average of Estimation Error Index 


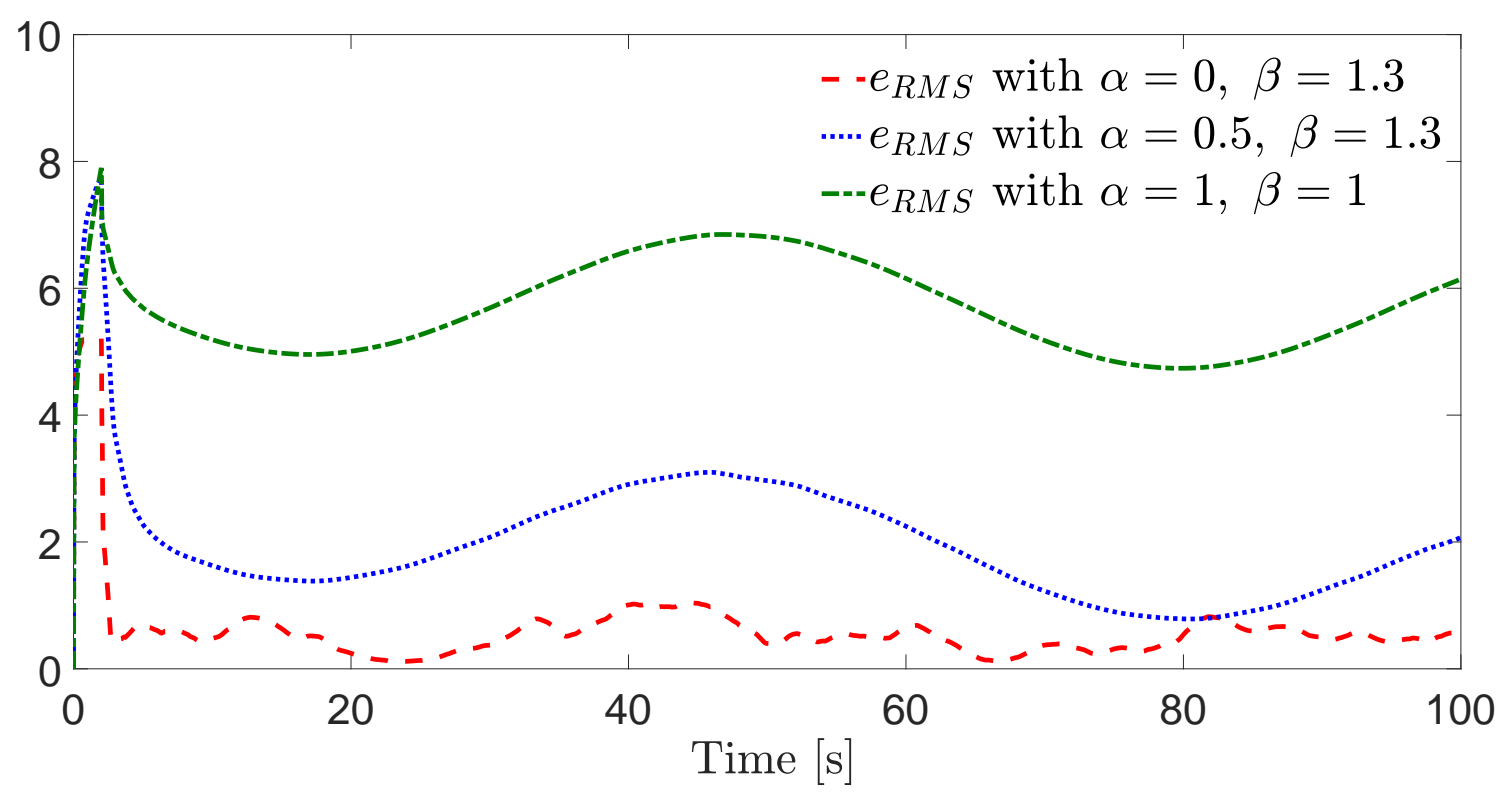

FIGURE 5 Estimation Error Index $e_{R M S}(t)$

\section{6 | CONCLUSIONS}

This paper contributes with a nonlinear adaptive sliding-mode observer based on a nonlinear time-varying parameter identification algorithm, for uncertain nonlinear systems with measurement noise. This nonlinear adaptive observer increases the class of nonlinear systems studied in ${ }^{20}$ considering unknown time-varying parameters, measurement noise, a parameter distribution matrix and nonlinear terms depending on the full state and the input. For the disturbed case, the nonlinear time-varying parameter identification algorithm provides a fixed-time rate of convergence while the sliding-mode observer ensures ultimate boundedness for the state estimation error attenuating the effects of the external disturbances. For the ideal case, i.e. without external disturbances and measurement noise, the adaptive observer provides finite-time convergence to a neighborhood of the origin for the state estimation error and fixed-time convergence to zero for the parameter identification error. The synthesis of the observer is given in terms of LMIs while the convergence proofs are developed based on Lyapunov and ISS theory. Some simulation results illustrate the performance of the proposed nonlinear adaptive sliding-mode observer.

\section{APPENDIX}

Proof of Lemma 3 Let us consider the following Lyapunov function:

$$
V_{\tilde{\theta}}=\frac{1}{2} \tilde{\theta}^{T} \Gamma^{-1} \tilde{\theta},
$$

that satisfies the following inequalities:

$$
\begin{gathered}
c_{1}^{-1}\|\tilde{\theta}\|^{2} \leq V_{\tilde{\theta}}(\tilde{\theta}) \leq c_{2}^{-1}\|\tilde{\theta}\|^{2}, \\
c_{1}^{-\frac{\alpha+1}{2}}\|\tilde{\theta}\|^{\alpha+1} \leq V_{\tilde{\theta}}^{\frac{\alpha+1}{2}}(\tilde{\theta}) \leq c_{2}^{-\frac{\alpha+1}{2}}\|\tilde{\theta}\|^{\alpha+1} .
\end{gathered}
$$

The time derivative of $V_{\tilde{\theta}}$ along the trajectories of the error dynamics $(12)$ is given by

$$
\dot{V}_{\tilde{\theta}}=-\tilde{\theta}^{T} \Gamma^{-1} \dot{\theta}-(C \Omega \tilde{\theta})^{T}\left[\lceil C \Omega \tilde{\theta}-C \delta-\varsigma\rfloor^{\alpha}+\lceil C \Omega \tilde{\theta}-C \delta-\varsigma]^{\beta}\right] .
$$

$\operatorname{In}^{32}$, it has been shown that the inequality

$$
x^{T}\lceil x+y\rfloor^{\alpha} \geq k_{1}\|x\|_{\alpha+1}^{\alpha+1}-k_{2}\|y\|_{\alpha+1}^{\alpha+1},
$$


holds for any $x, y \in \mathbb{R}^{n}, \alpha>0, k_{1} \in(0,1)$ and $k_{2}=\max \left(k_{1}+1, k_{1} /\left(1-k_{1}^{\frac{1}{\alpha}}\right)^{\alpha}\right)$. Then, applying the previous inequality, one obtains

$$
\dot{V}_{\tilde{\theta}} \leq-k_{11}\|C \Omega \tilde{\theta}\|_{\alpha+1}^{\alpha+1}-k_{12}\|C \Omega \tilde{\theta}\|_{\beta+1}^{\beta+1}+k_{21}\|C \delta+\varsigma\|_{\alpha+1}^{\alpha+1}+k_{22}\|C \delta+\varsigma\|_{\beta+1}^{\beta+1}+\left\|\tilde{\theta}^{T} \Gamma^{-1} \dot{\theta}\right\| .
$$

where $k_{11}, k_{12} \in(0,1), k_{21}=\max \left(k_{11}+1, k_{11} /\left(1-k_{11}^{\frac{1}{\alpha}}\right)^{\alpha}\right)$ and $k_{22}=\max \left(k_{12}+1, k_{12} /\left(1-k_{12}^{\frac{1}{\beta}}\right)^{\beta}\right)$. By the relations between vector norms and by Holder's inequality, one has that $\|C \Omega \tilde{\theta}\| \leq\|C \Omega \tilde{\theta}\|_{\alpha+1},\|C \Omega \tilde{\theta}\| \leq p^{\frac{\beta-1}{2(\beta+1)}}\|C \Omega \tilde{\theta}\|_{\beta+1},\|C \delta+\varsigma\|_{\alpha+1} \leq$ $p^{\frac{1}{\alpha+1}}\|C \delta+\varsigma\|,\|C \delta+\varsigma\|_{\beta+1} \leq\|C \delta+\varsigma\|$, with $p$ being the dimension of the output, hold since $\beta+1>2>\alpha+1$. Hence, taking into account assumptions 2 and 3 , it follows that

$$
\dot{V}_{\tilde{\theta}} \leq-k_{11} \varrho_{\min }^{\alpha+1}\|\tilde{\theta}\|^{\alpha+1}-p^{\frac{1-\beta}{2(\beta+1)}} k_{12} \varrho_{\min }^{\beta+1}\|\tilde{\theta}\|^{\beta+1}+p k_{21}\|C \delta+\varsigma\|^{\alpha+1}+k_{22}\|C \delta+\varsigma\|^{\beta+1}+2 c_{2}^{-1} \bar{\theta}\|\tilde{\theta}\| .
$$

Then, $\dot{V}_{\tilde{\theta}}$ can be upper bounded as

$$
\begin{aligned}
\dot{V}_{\tilde{\theta}} \leq-k_{11}\left(1-\varphi_{1}\right) \rho_{\min }^{\alpha+1}\|\tilde{\theta}\|^{\alpha+1}-p^{\frac{1-\beta}{2(\beta+1)}} k_{12}\left(1-\varphi_{2}\right) \varrho_{\min }^{\beta+1}\|\tilde{\theta}\|^{\beta+1}-\varphi_{1} k_{11} \varrho_{\min }^{\alpha+1}\|\tilde{\theta}\|^{\alpha+1}-p^{\frac{1-\beta}{2(\beta+1)}} \varphi_{2} k_{12} \varrho_{\min }^{\beta+1}\|\tilde{\theta}\| \|^{\beta+1} \\
+p k_{21}\|C \delta+\varsigma\|^{\alpha+1}+k_{22}\|C \delta+\varsigma\|^{\beta+1}+2 c_{2}^{-1} \bar{\theta}\|\tilde{\theta}\|,
\end{aligned}
$$

for any constants $\varphi_{1}, \varphi_{2} \in(0,1)$. Therefore, it is given that

$$
\begin{gathered}
\dot{V}_{\tilde{\theta}} \leq-k_{11}\left(1-\varphi_{1}\right) \rho_{\min }^{\alpha+1} c_{2}^{\frac{\alpha+1}{2}} V_{\tilde{\theta}}^{\frac{\alpha+1}{2}}-p^{\frac{1-\beta}{2(\beta+1}} k_{12}\left(1-\varphi_{2}\right) \rho_{\min }^{\beta+1} c_{2}^{\frac{\beta+1}{2}} V_{\tilde{\theta}}^{\frac{\beta+1}{2}}, \forall\|\tilde{\theta}\| \geq \max \left[\frac{\left(2 p k_{21}\right)^{\frac{1}{\alpha+1}}}{\left(\varphi_{1} k_{11}\right)^{\frac{1}{\alpha+1}} \rho_{\min }}\left(\|C\|\|\delta\|_{\infty}+\|\varsigma\|_{\infty}\right)+\kappa_{\tilde{\theta} 1},\right. \\
\left.\frac{\left(2 k_{22}\right)^{\frac{1}{\beta+1}}}{\left(p^{\frac{1-\beta}{2(\beta+1}} \varphi k_{12}\right)^{\frac{1}{\beta+1}} \rho_{\min }}\left(\|C\|\|\delta\|_{\infty}+\|\varsigma\|_{\infty}\right)\right] \text {, with } \kappa_{\tilde{\theta} 1}=\left(\frac{4 c_{2}^{-1} \bar{\theta}}{\varphi k_{11} \rho_{\min }^{\alpha+1}}\right)^{\frac{1}{\alpha}} .
\end{gathered}
$$

Then, the error dynamics (12) is ISpS with respect to the inputs $\delta$ and $\varsigma$, and applying Lemma 2 , its trajectories satisfy the bounds given by (14)-(16).

Before providing the proof of Lemma4 some inequalities are introduced.

Lemma 6. Let $x, \delta \in \mathbb{R}$. Then, for any $k_{1} \in(0,1)$ and $k_{2} \geq k_{1}+1$

$$
x\lceil x+\delta\rfloor^{0} \geq k_{1}|x|-k_{2}|\delta| .
$$

Proof of Lemma 6. For the case $x=0$ or $\delta=0$, A.28) trivially holds with any $k_{2} \geq 0$ and $k_{1} \in(0,1)$, respectively. For the case $x \neq 0$ or $\delta \neq 0$, if $|x| \geq|\delta|$, then $\lceil x+\delta\rfloor^{0}=\operatorname{sign}(x)$. Consequently, $x\lceil x+\delta\rfloor^{0}=|x|$ and the needed property is also valid for any $k_{1} \in(0,1)$ and $k_{2} \geq 0$. Finally, if $|x|<|\delta|$, then $\lceil x+\delta\rfloor^{0}=\operatorname{sign}(\delta)$ and $x\lceil x+\delta\rfloor^{0} \geq-|\delta|$. Thus, the required inequality holds for any $k_{2} \geq k_{1}+1$ and any $k_{1} \in(0,1)$. This concludes the proof.

Lemma 7. Let $x, \delta \in \mathbb{R}^{n}$. Then, for any $k_{1} \in(0,1)$ and $k_{2} \geq k_{1}+1$

$$
x^{T}\lceil x+\delta\rfloor^{0} \geq k_{1}\|x\|-k_{2} \sqrt{n}\|\delta\| .
$$

Proof of Lemma 7. The proof is straightforward from Lemma 6 and the equivalence between norms.

Proof of Lemma 4 Let us consider the Lyapunov function given in A.24) that satisfies the inequalities A.25, and A.26. The time derivative of $V_{\tilde{\theta}}$ along the trajectories of the error dynamics $(12)$, for $\alpha=0$, is given by

$$
\dot{V}_{\tilde{\theta}}=-(C \Omega \tilde{\theta})^{T}\left[\lceil C \Omega \tilde{\theta}-C \delta-\varsigma\rfloor^{0}+[C \Omega \tilde{\theta}-C \delta-\varsigma\rfloor^{\beta}\right]-\tilde{\theta}^{T} \Gamma^{-1} \dot{\theta} .
$$

Applying the inequality A.29, given in Lemma 7 Holder's inequality, and inequality (A.27), it follows that

$$
\dot{V}_{\tilde{\theta}} \leq-k_{11}\|C \Omega \tilde{\theta}\|-p^{\frac{1-\beta}{2(\beta+1)}} k_{12}\|C \Omega \tilde{\theta}\|^{\beta+1}+\sqrt{p} k_{21}\|C \delta+\varsigma\|+k_{22}\|C \delta+\varsigma\|^{\beta+1}+\left\|\tilde{\theta}^{T} \Gamma^{-1} \dot{\theta}\right\|,
$$

where $k_{11}, k_{12} \in(0,1), k_{21} \geq k_{11}+1$ and $k_{22}=\max \left(k_{12}+1, k_{12} / 1-k_{12}^{\frac{1}{\beta}}\right)^{\beta}$. Hence, by assumptions 2 and 3 . it follows that

$$
\dot{V}_{\tilde{\theta}} \leq-k_{11} \varrho_{\min }\|\tilde{\theta}\|-p^{\frac{1-\beta}{2(\beta+1)}} k_{12} \varrho_{\min }^{\beta+1}\|\tilde{\theta}\|^{\beta+1}+\sqrt{p} k_{21}\|C \delta+\varsigma\|+k_{22}\|C \delta+\varsigma\|^{\beta+1}+2 c_{2}^{-1} \bar{\theta}\|\tilde{\theta}\| .
$$


Thus, $\dot{V}_{\tilde{\theta}}$ can be upper bounded as

$$
\begin{aligned}
\dot{V}_{\tilde{\theta}} \leq-k_{11}\left(1-\varphi_{1}\right) \varrho_{\min }\|\tilde{\theta}\|-p^{\frac{1-\beta}{2(\beta+1)}} k_{12}\left(1-\varphi_{2}\right) \varrho_{\min }^{\beta+1}\|\tilde{\theta}\|^{\beta+1}-\varphi_{1} k_{11} \varrho_{\min }\|\tilde{\theta}\|-\varphi_{2} p^{\frac{1-\beta}{2(\beta+1)}} k_{12} \varrho_{\min }^{\beta+1}\|\tilde{\theta}\|^{\beta+1} \\
+\sqrt{p} k_{21}\|C \delta+\varsigma\|+k_{22}\|C \delta+\varsigma\|^{\beta+1}+2 c_{2}^{-1} \bar{\theta}\|\tilde{\theta}\|
\end{aligned}
$$

for any constant $\varphi_{1}, \varphi_{2} \in(0,1)$. Hence, it is obtained that

$$
\begin{array}{r}
\dot{V}_{\tilde{\theta}} \leq-k_{11}\left(1-\varphi_{1}\right) \varrho_{\min } c_{2}^{\frac{1}{2}} V_{\tilde{\theta}}^{\frac{1}{2}}-p^{\frac{1-\beta}{2(\beta+1)}} k_{12}\left(1-\varphi_{2}\right) \rho_{\min }^{\beta+1} c_{2}^{\frac{\beta+1}{2}} V_{\tilde{\theta}}^{\frac{\beta+1}{2}}, \forall\|\tilde{\theta}\| \geq \max \\
{\left[\frac{2 \sqrt{p} k_{21}}{\varphi_{1} k_{11} \rho_{\min }}\left(\|C\|\|\delta\|_{\infty}+\|\varsigma\|_{\infty}\right),\right.} \\
\left.\frac{\left(2 k_{22}\right)^{\frac{1}{\beta+1}}}{\left(p^{\frac{1-\beta}{2(\beta+1)}} \varphi k_{12}\right)^{\frac{1}{\beta+1}} \varrho_{\min }}\left(\|C\|\|\delta\|\left\|_{\infty}+\right\| \varsigma \|_{\infty}\right)\right],
\end{array}
$$

for any $\lambda_{\min }(\Gamma) \geq 2 \bar{\theta} / \varphi k_{11} \rho_{\min }$. Then, the error dynamics $(12)$, for $\alpha=0$, is ISS with respect to the inputs $\delta$ and $\varsigma$, and applying Lemma 2 its trajectories satisfy the bounds given by (17)-(19).

Proof of Lemma 5. Let us consider the following candidate Lyapunov function:

$$
V_{\delta}=\delta^{T} P \delta,
$$

that satisfies the following inequality:

$$
r_{1}\|\delta\|^{2} \leq V_{\delta} \leq r_{2}\|\delta\|^{2},
$$

with $r_{1}=\lambda_{\min }(P)$ and $r_{2}=\lambda_{\max }(P)$. The time derivative of $V_{\delta}$ along the trajectories of the error dynamics (13) is given by $\dot{V}_{\delta}=\delta^{T}\left[P(A-L C)+P(A-L C)^{T}\right] \delta+2 \delta^{T} P D(w-k \operatorname{sign}[F(y-C \hat{x})])+2 \delta^{T} P\left(\Delta_{\phi}(x, \hat{x}, u)+\Delta_{G}(t, x, \hat{x}, u) \theta\right)-2 \delta P(\Omega \dot{\theta}+L \varsigma)$.

Then, using (20) together with Schur's complement, one obtains

$$
\begin{gathered}
\dot{V}_{\delta} \leq-\delta^{T}\left(\vartheta P+\sigma C^{T} C+P \eta_{1}+\varepsilon^{-1} \eta_{2}^{2} P^{2}\right) \delta+2 \delta^{T} C^{T} F^{T}(w-k \operatorname{sign}[F(y-C \hat{x})]) \\
+2 \delta^{T} P\left(\Delta_{\phi}(x, \hat{x}, u)+\Delta_{G}(t, x, \hat{x}, u) \theta\right)-2 \delta P(\Omega \dot{\theta}+L \varsigma) .
\end{gathered}
$$

Recalling that $\delta=x-\hat{x}+\Omega \tilde{\theta}$, it follows that $C \delta=y-C \hat{x}+C \Omega \tilde{\theta}$, and thus

$$
\begin{gathered}
\dot{V}_{\delta} \leq-\delta^{T}\left(\vartheta P+\sigma C^{T} C+P \eta_{1}+\varepsilon^{-1} \eta_{2}^{2} P^{2}\right) \delta+2(y-C \hat{x})^{T} F^{T}(w-k \operatorname{sign}[F(y-C \hat{x})]) \\
+2 \tilde{\theta}^{T} \Omega^{T} C^{T} F^{T}(w-k \operatorname{sign}[F(y-C \hat{x})])+2 \delta^{T} P\left(\Delta_{\phi}(x, \hat{x}, u)+\Delta_{G}(t, x, \hat{x}, u) \theta\right)-2 \delta P(\Omega \dot{\theta}+L \varsigma) .
\end{gathered}
$$

Fixing $k=\|\omega\|_{\infty}$, the derivative of $V_{\delta}$ can be upper bounded as follows

$$
\dot{V}_{\delta} \leq-\delta^{T}\left(\vartheta P+\sigma C^{T} C+P \eta_{1}+\varepsilon^{-1} \eta_{2}^{2} P^{2}\right) \delta+4\|C \Omega \tilde{\theta}\|\|\mid F\|\|w\|_{\infty}+2 \delta^{T} P\left(\Delta_{\phi}(x, \hat{x}, u)+\Delta_{G}(t, x, \hat{x}, u) \theta\right)-2 \delta P(\Omega \dot{\theta}+L \varsigma) .
$$

By Assumption 2, one gets that the following inequality holds $\left\|\Delta_{\phi}+\Delta_{G} \theta\right\| \leq\left(L_{\phi}+L_{G} \theta^{+}\right)\left(\|\delta\|_{\infty}+\|\tilde{\theta}\|\|\Omega\| \|_{\infty}\right)$. Therefore, the derivative of $V_{\delta}$ is upper bounded as

$$
\begin{gathered}
\dot{V}_{\delta} \leq-\delta^{T}\left(\vartheta P+\sigma C^{T} C+P \eta_{1}+\varepsilon^{-1} \eta_{2}^{2} P^{2}\right) \delta+4\|C \Omega \tilde{\theta}\|\|F\|\|w\|_{\infty}+2 \delta^{T} P\left(L_{\phi}+L_{G} \theta^{+}\right) \delta \\
+2 \delta^{T} P\left(L_{\phi}+L_{G} \theta^{+}\right)\|\tilde{\theta}\| \varrho_{\max }\|C\|^{-1}+2 r_{2}\left(\bar{\theta} \varrho_{\max }\|C\|^{-1}+\|\varsigma\|\|L\|\right)\|\delta\| .
\end{gathered}
$$

Recalling that $\eta_{1}=2\left(L_{\phi}+L_{G} \theta^{+}\right)$and $\eta_{2}=\eta_{1} \varrho_{\max }\|C\|^{-1}$, it follows that

$$
\dot{V}_{\delta} \leq-\delta^{T}\left(\vartheta P+\sigma C^{T} C+\varepsilon^{-1} \eta_{2}^{2} P^{2}\right) \delta+4\|C \Omega \tilde{\theta}\|\||| F \mid\|\|w\|_{\infty}+\delta^{T} P \eta_{2}\|\tilde{\theta}\|+2 r_{2}\left(\bar{\theta} \varrho_{\max }\|C\|^{-1}+\|\varsigma\|\|L\|\right)\|\delta\| .
$$

It is well-known that the inequality $\delta^{T} P \eta_{2}\|\tilde{\theta}\| \leq \varepsilon^{-1} \eta_{2}^{2} \delta^{T} P^{2} \delta+\varepsilon\|\tilde{\theta}\|^{2}$ holds for any $\varepsilon>0$. Thus, the previous inequality is upper bounded as follows

$$
\dot{V}_{\delta} \leq-\delta^{T}\left(\vartheta P+\sigma C^{T} C\right) \delta+4\|C \Omega \tilde{\theta}\|\|F\|\|\mid w\|_{\infty}+\varepsilon\|\tilde{\theta}\|^{2}+2 r_{2}\left(\bar{\theta} \varrho_{\max }\|C\|^{-1}+\|\varsigma\|\|L\|\right)\|\delta\| .
$$

That can be rewritten as

$$
\begin{gathered}
\dot{V}_{\delta} \leq-\delta^{T}\left(\frac{\vartheta}{2} P+\frac{1}{2} \sigma C^{T} C\right) \delta+4\|C \Omega \tilde{\theta}\|\left\|| | F \left|\||| w\|_{\infty}+\varepsilon\|\tilde{\theta}\|^{2}\right.\right. \\
-\frac{1}{2} \sigma\|y-C \hat{x}\|^{2}-\frac{1}{2} \sigma\|C \Omega \tilde{\theta}\|^{2}+\sigma\|y-C \hat{x}\|\|C \Omega \tilde{\theta}\|+2 r_{2}\left(\bar{\theta} \varrho_{\max }\|C\|^{-1}+\|\varsigma\|\|L\|\right)\|\delta\|-\frac{\vartheta}{2} r_{1}\|\delta\|^{2} .
\end{gathered}
$$


Taking into account that

$$
\begin{gathered}
-\frac{1}{2} \sigma\|C \Omega \tilde{\theta}\|^{2}+4\|C \Omega \tilde{\theta}\|\|F\|\|w\|_{\infty} \leq 8 \sigma^{-1}\|F\|^{2}\|w\|_{\infty}^{2}, \\
-\frac{1}{2} \sigma\|y-C \hat{x}\|^{2}+\sigma\|y-C \hat{x}\|\|C \Omega \tilde{\theta}\| \leq \frac{1}{2} \sigma\|C \Omega \tilde{\theta}\|^{2}, \\
-\frac{\vartheta}{2} r_{1}\|\delta\|^{2}+2 r_{2}\left(\bar{\theta} \varrho_{\max }\|C\|^{-1}+\|\varsigma\|\|L\|\right)\|\delta\| \leq 2 \vartheta^{-1} r_{1}^{-1} r_{2}^{2}\left(\bar{\theta} \varrho_{\max }\|C\|^{-1}+\|\varsigma\|\|L\|\right)^{2},
\end{gathered}
$$

one obtains that

$$
\dot{V}_{\delta} \leq-\alpha_{\delta}\|\delta\|^{2}+\left(\varepsilon+\frac{1}{2} \sigma \varrho_{\max }^{2}\right)\|\tilde{\theta}\|^{2}+8 \sigma^{-1}\|F\|^{2}\|w\|_{\infty}^{2}+2 \vartheta^{-1} r_{1}^{-1} r_{2}^{2}\left(\bar{\theta} \varrho_{\max }\|C\|^{-1}+\|\varsigma\|\|L\|\right)^{2},
$$

where $\alpha_{\delta}=\left(\vartheta r_{1}+\sigma\|C\|^{2}\right) / 2$. Hence, for all $\rho \in(0,1)$, it is given that

$$
\begin{gathered}
\dot{V}_{\delta} \leq-\frac{(1-\rho) \alpha_{\delta}}{r_{2}} V_{\delta}, \forall\|\delta\| \geq \sqrt{\frac{2 \varepsilon+\sigma \varrho_{\max }^{2}}{2 \rho \alpha_{\delta}}}\|\tilde{\theta}\|_{\infty}+\sqrt{\frac{2 r_{2}^{2}}{\vartheta r_{1} \rho \alpha_{\delta}}}\|\varsigma\|_{\infty}\|L\|+\sqrt{\frac{8\|F\|^{2}}{\rho \alpha_{\delta} \sigma}}\|w\|_{\infty}+\kappa_{\delta}, \\
\kappa_{\delta}=\sqrt{\frac{2 r_{2}^{2}}{\vartheta r_{1} \rho \alpha_{\delta}}}\left(\bar{\theta} \varrho_{\max }\|C\|^{-1}\right) .
\end{gathered}
$$

Thus the error dynamics (13) is ISpS with respect to the inputs $\tilde{\theta}$ and $w$, and applying Lemma 1 , its trajectories satisfy the bounds given by $(21)$ and $(\overline{22})$.

Proof of Theorem 2 Consider the Lyapunov functions $V_{\tilde{\theta}}=\frac{1}{2} \tilde{\theta}^{T} \Gamma^{-1} \tilde{\theta}$ and $V_{\delta}=\delta^{T} P \delta$. Taking into account the statements given in lemmas 3 and 5 , it follows that

$$
\begin{gathered}
\dot{V}_{\tilde{\theta}} \leq-k_{11}\left(1-\varphi_{1}\right) \varrho_{\min }^{\alpha+1} c_{2}^{\frac{\alpha+1}{2}} V_{\tilde{\theta}}^{\frac{\alpha+1}{2}}, \forall V_{\tilde{\theta}} \geq \max \left[\frac{2\left(2 p k_{2}\right)^{\frac{2}{\alpha+1}}\|C\|^{2}}{\left(\varphi_{1} k_{11}\right)^{\frac{2}{\alpha+1}} \rho_{\min }^{2} r_{1} c_{2}} V_{\delta}, 2 \bar{\kappa}_{\tilde{\theta} 1}(\|\varsigma\|)\right], \\
\bar{\kappa}_{\tilde{\theta} 1}(\|\varsigma\|)=\frac{\left(2 p k_{2}\right)^{\frac{2}{\alpha+1}}}{\left(\varphi_{1} k_{11}\right)^{\frac{2}{\alpha+1}} \rho_{\min }^{2} c_{2}}\|\varsigma\|^{2}+\frac{\kappa_{\tilde{\theta} 1}}{c_{2}}
\end{gathered}
$$

and

$$
\begin{gathered}
\dot{V}_{\delta} \leq-\frac{(1-\rho) \alpha_{\delta}}{r_{2}} V_{\delta}, \forall V_{\delta} \geq \max \left[\frac{2 c_{1}\left(2 \varepsilon+\sigma \rho_{\max }^{2}\right)}{\rho \alpha_{\delta} r_{2}} V_{\tilde{\theta}}, \bar{\kappa}_{\delta}(\|w\|,\|\varsigma\|)\right], \\
\bar{\kappa}_{\delta}(\|w\|,\|\varsigma\|)=\frac{32\|F\|^{2}}{\rho \alpha_{\delta} \sigma r_{2}}\|w\|^{2}+\frac{8 r_{2}\|L\|^{2}}{\vartheta r_{1} \rho \alpha_{\delta}}\|\varsigma\|^{2}+\frac{4 \kappa_{\delta}^{2}}{r_{2}} .
\end{gathered}
$$

Thus, according to Theorem 11, one can define

$$
\begin{gathered}
\chi_{1}\left(V_{\delta}\right)=\frac{2\left(2 p k_{21}\right)^{\frac{2}{\alpha+1}}\|C\|^{2}}{\left(\varphi_{1} k_{11}\right)^{\frac{2}{\alpha+1}} \rho_{\min }^{2} r_{1} c_{2}} V_{\tilde{\theta}}, \\
\chi_{2}\left(V_{\tilde{\theta}}\right)=\frac{2 c_{1}\left(2 \varepsilon+\sigma \rho_{\max }^{2}\right)}{\rho \alpha_{\delta} r_{2}} V_{\tilde{\theta}} .
\end{gathered}
$$

Hence, it follows that

$$
\chi_{1} \circ \chi_{2}(r)=\left[\frac{4 c_{1}\left(2 p k_{21}\right)^{\frac{2}{\alpha+1}}\|C\|^{2}\left(2 \varepsilon+\sigma \rho_{\max }^{2}\right)}{\left(\varphi_{1} k_{11}\right)^{\frac{2}{\alpha+1}} \rho_{\min }^{2} \rho \alpha_{\delta} r_{1} r_{2} c_{2}}\right] r .
$$

Therefore, if the matrix inequalities $(20)$ hold and the following inequality

$$
\frac{4 \lambda_{\max }(\Gamma)\left(2 p k_{21}\right)^{\frac{2}{\alpha+1}}\|C\|^{2}\left(2 \varepsilon+\sigma \varrho_{\max }^{2}\right)}{\left(\varphi_{1} k_{11}\right)^{\frac{2}{\alpha+1}} \varrho_{\min }^{2} \rho \alpha_{\delta} r_{1} r_{2} \lambda_{\min }(\Gamma)}<1,
$$

is satisfied, based on lemmas 3 and 5 , and Theorem 1 , one can conclude that the interconnected error system (12)-(13) is ISpS with respect to the external inputs $w$ and $\varsigma$ for any $\alpha \in(0,1)$.

Note that satisfaction of (A.32) can be always guaranteed by a proper adjustment of the parameters. Indeed, assume that the matrix inequalities $(20)$ are feasible and the set of all matrices and parameters from lemmas $3-5$ is given, but the inequality 
A.32) fails to be correct. Then one can just scale the value of the matrix $P$ in $(20)$, i.e. $P \rightarrow \imath P$ for some $\imath>1$ (hence, $r_{1}$ and $r_{2}$ are also increased by $l$ ). In such a case the matrix $L$ stays the same but, $\Lambda, \epsilon, \sigma$ and $\vartheta$ have to be also scaled by $l$ ( $\alpha_{\delta}$ grows quadratically in $\imath$ ). The constants $k_{11}, k_{21}, \rho$ and $\varphi_{1}$ stay unchanged. Therefore, the denominator of (A.32) is growing as $\imath^{4}$ and numerator proportionally to $l$, then there is a value of $\iota$ such that the inequality $(\mathrm{A} .32)$ is satisfied for any matrix $\Gamma$.

Proof of Theorem 3 Considering the statements given in lemmas 4 and 5 it follows that

$$
\begin{gathered}
\dot{V}_{\tilde{\theta}} \leq-k_{11}\left(1-\varphi_{1}\right) \varrho_{\min } c_{2}^{\frac{1}{2}} V_{\tilde{\theta}}^{\frac{1}{2}}, \forall V_{\tilde{\theta}} \geq \max \left[\frac{8 p k_{21}^{2}\|C\|^{2}}{\varphi_{1}^{2} k_{11}^{2} \rho_{\min }^{2} r_{1} c_{2}} V_{\delta}, \kappa_{\tilde{\theta} 2}(\|\varsigma\|)\right], \\
\kappa_{\tilde{\theta} 2}(\|\varsigma\|)=\frac{8 p k_{2}^{2}}{\varphi_{1}^{2} k_{11}^{2} \rho_{\min }^{2} c_{2}}\|\varsigma\|^{2},
\end{gathered}
$$

and

$$
\begin{gathered}
\dot{V}_{\delta} \leq-\frac{(1-\rho) \alpha_{\delta}}{r_{2}} V_{\delta}, \forall V_{\delta} \geq \max \left[\frac{2 c_{1}\left(2 \varepsilon+\sigma \rho_{\max }^{2}\right)}{\rho \alpha_{\delta} r_{2}} V_{\tilde{\theta}}, \bar{\kappa}_{\delta}(\|w\|,\|\varsigma\|)\right], \\
\bar{\kappa}_{\delta}(\|w\|,\|\varsigma\|)=\frac{32\|F\|^{2}}{\rho \alpha_{\delta} \sigma r_{2}}\|w\|^{2}+\frac{8 r_{2}\|L\|^{2}}{\vartheta r_{1} \rho \alpha_{\delta}}\|\varsigma\|^{2}+\frac{4 \kappa_{\delta}^{2}}{r_{2}} .
\end{gathered}
$$

Hence, according to Theorem 1 , one can define

$$
\begin{gathered}
\chi_{1}\left(V_{\delta}\right)=\frac{8 p k_{21}^{2}\|C\|^{2}}{\varphi_{1}^{2} k_{11}^{2} \rho_{\min }^{2} r_{1} c_{2}} V_{\delta}, \\
\chi_{2}\left(V_{\tilde{\theta}}\right)=\frac{2 c_{1}\left(2 \varepsilon+\sigma \varrho_{\max }^{2}\right)}{\rho \alpha_{\delta} r_{2}} V_{\tilde{\theta}} .
\end{gathered}
$$

Thus, it is obtained that

$$
\chi_{1} \circ \chi_{2}(r)=\left[\frac{16 c_{1} p k_{21}^{2}\|C\|^{2}\left(2 \varepsilon+\sigma \varrho_{\max }^{2}\right)}{\varphi_{1}^{2} k_{11}^{2} \rho_{\min }^{2} \rho \alpha_{\delta} r_{1} r_{2} c_{2}}\right] r .
$$

Therefore, if the matrix inequalities (20) hold and the following inequality

$$
\frac{16 \lambda_{\max }(\Gamma) p k_{21}^{2}\|C\|^{2}\left(2 \varepsilon+\sigma \varrho_{\max }^{2}\right)}{\varphi_{1}^{2} k_{11}^{2} \rho_{\min }^{2} \rho \alpha_{\delta} r_{1} r_{2} \lambda_{\min }(\Gamma)}<1,
$$

is satisfied, based on lemmas 4 and 5 and Theorem 1 , one concludes that the interconnected error system (12)-(13) is ISpS with respect to the external inputs $w$ and $\varsigma$ for $\alpha=0$.

Following the same arguments as in proof of Theorem 2, the denominator of (A.33) grows as $\imath^{4}$ and numerator does proportionally to $l$, then there is always a value of $\iota$ such that A.33 is also satisfied for any matrix $\Gamma$. Therefore, A.33 is always guaranteed.

\section{ACKNOWLEDGMENTS}

R. Franco and H. Ríos thank the financial support from CONACYT CVU 772057, and Cátedras CONACYT CVU 270504 and project 922, respectively; and from TECNM projects.

\section{References}

1. Franco R, Ríos H, Efimov D, Perruquetti W. Adaptive estimation for uncertain nonlinear systems: A sliding-mode observer approach. In: Proceedings of the 57th IEEE Conference on Decision and Control. CDC 2018. ; 2018; Miami Beach, FL, USA. pp. 5506-5511.

2. Astolfi A, Karaglannis D, Ortega R. Nonlinear and Adaptive Control with Applications. Springer-Verlang London UK . 2008. 
3. Krstic M, Kanellakopoulos I, Kokotovic P. Nonlinear and Adaptive Control Design. Canada: Wiley-Interscience . 1995.

4. Besançon G. Nonlinear Observers and Application. Springer-Verlang Berlin Heidelberg . 2007.

5. Pu Z, Yuan R, Yi J, Tan X. A class of adaptive extended state observers for nonlinear disturbed systems. IEEE Transactions on Industrial Electronics 2015; 62: 5858-5869.

6. Ekramian M, Sheikholeslam F, Hosseinnia S, Yazdanpanah M. Adaptive state observer for Lipschitz nonlinear systems. Systems \& Control Letters 2013; 62: 319-323.

7. Farza M, M Saad M, Maatoug T, Kamoun M. Adaptive observers for nonlinear parameterized class of nonlinear systems. Automatica. 2009; 45: 2292-2299.

8. Tyukin I, Steur E, Neijmeijer H, Leuwen C. Adaptive observers and parameter estimation for a class of systems nonlinear in the parameters. Automatica 2013; 49: 2409.2423.

9. Oyvind N, Morten AO, Glenn-Ole K. Redesign of adaptive observers for improved parameter identification in nonlinear systems. Automatica 2010; 47: 403-410.

10. Ding F, Ding J. Least-squares parameter estimation for systems with irregularly missing data. International Journal of Adaptive Control and Signal Processing 2010; 24: 540-553.

11. Stepanyan V, Hovakimyan N. Robust Adaptive Observer Design for Uncertain Systems with Bounded Disturbances. IEEE Transactions on Neural Networks 2007; 18: 1392-1403.

12. Jiang J, Zhang Y. A novel variable-length sliding window blockwise least-squares algorithm for on-line estimation of timevarying parameters. International Journal of Adaptive Control and Signal Processing 2004; 18: 505-521.

13. Ríos H, Efimov D, Moreno J, Perruquetti W, Rueda-Escobedo J. Time-Varying Parameter Identification Algorithms: Finite and Fixed-Time Convergence. IEEE Transactions on Automatic Control 2017; 62: 3671-3678.

14. Zhang H, Wang J. Adaptive sliding-mode observer design for a selective catalytic reduction system of ground-vehicle diesel engines. IEEE Transactions on Mechatronics 2016; 21: 2027-2038.

15. Rahme S, Meskin N. Adaptive sliding-mode observer for sensor fault diagnosis of an industrial gas turbine. Control Engineering Practice 2015; 38: 57-74.

16. Laghrouche S, Liu J, Ahmed S, Harmouche M, Wack M. Adaptive second-order sliding-mode observer-based fault reconstrucion for PEM fuel cell air-feed system. IEEE Transactions on Control Systems Technology 2015; 23: 1098-1109.

17. Yan X, Edwards C. Adaptive sliding-mode-observer-based fault reconstruction for nonlineary systems with parametric unvertainties. IEEE Transactions on Industrial Electronics 2008; 55: 4029-4036.

18. Farza M, Saad M, Menard T, Ltaief TA, Maatoug T. Adaptive Observer Design for a Class of Nonlinear Systems. Application to Speed Sesnorless Induction Motor. Automatica 2018; 90: 239-247.

19. Efimov D, Fradkov A. Design of impulsive adaptive observers for improvement of persistency of excitation. International Journal of Adaptive Control Signal 2015; 29: 765-782.

20. Ríos H, Efimov D, Perruquetti W. An adaptive sliding-mode observer for a class of uncertain nonlinear systems. International Journal of Adaptive Control and Signal Processing 2018; 32: 511-527.

21. Bernuau E, Polyakov A, Efimov D, Perruquetti W. Verification of ISS, iISS and IOSS properties applying weighted homogeneity. Systems \& Control Letters 2013; 62: 1159-1167.

22. Jiang Z, Mareels I, Wang Y. A Lyapunov formulation of the nonlinear small-gain theorem for interconnected ISS systems. Automatica 1996; 32: 1211-1215.

23. Sontag ED. On the Input-to-State Stability Property. European Journal of Control 1995; 1(1): 24-36. 
24. Khalil HK. Nonlinear Systems. Prentice-Hall, Upsaddle River, New Jersey 3rd. edition . 2002.

25. Polyakov A. Nonlinear feedback design for fixed-time stabilization of linear control systems. IEEE Transactions on Automatic Control 2012; 57(8): 2106-2110.

26. Danca M, Codreanu S. On a possible approximation of discontinuous dynamical systems. Chaos, Solutions and Fractals 2002; 13: 681-691.

27. Filippov AF. Differential Equations with Discontinuous Righthand side. Mathematics and its Applications (Soviet Series). Kluwer, Dordrecht, The Netherlands . 1988.

28. Bernuau E, Efimov D, Perruquetti W, Polyakov A. On homogeneity and its application in sliding-mode. International Journal of Franklin Institute 2014; 351: 1866-1901.

29. Rueda-Escobedo J, Moreno J, Schiffer J. Discontinuous gradient algorithm for finite-time estimation of time-varying parameters. International Journal of Control 2016; 89: 1838-1848.

30. Poznyak AS. Advanced Mathematical Tools for Automatic Control Engineers. Vol 1: Deterministic Techniques. Amsterdam: Elsevier . 2008.

31. Huang C, Chen Y. Adaptive Sliding Control for Single-Link Flexible-Joint Robot With Mismatched Uncertainties. IEEE Transactions on Control Systems Technology 2004; 12: 770-775.

32. Rueda-Escobedo J, Ushirobira R, Efimov D, Moreno J. Gramian-based uniform convergent observer for stable LTV systems. International Journal of Control 2019. doi: 10.1080/00207179.2019.1569256. 